\title{
DISKS IN THE ARCHES CLUSTER—SURVIVAL IN A STARBURST ENVIRONMENT*
}

\author{
A. Stolte $^{1,2}$, M. R. Morris ${ }^{2}$, A. M. Ghez ${ }^{2,3}$, T. Do ${ }^{2}$, J. R. Lu ${ }^{4}$, S. A. Wright ${ }^{5}$, C. Ballard ${ }^{6}$, E. Mills ${ }^{2}$, And K. Matthews ${ }^{4}$ \\ ${ }^{1}$ I. Physikalisches Institut, Universität zu Köln, Zülpicher Str. 77, 50937 Köln, Germany; astolte@ph1.uni-koeln.de \\ ${ }^{2}$ Division of Astronomy and Astrophysics, UCLA, Los Angeles, CA 90095-1547, USA; ghez@astro.ucla.edu, morris@astro.ucla.edu, jlu@astro.ucla.edu \\ ${ }^{3}$ Institute of Geophysics and Planetary Physics, UCLA, Los Angeles, CA 90095, USA \\ ${ }^{4}$ Caltech Optical Observatories, California Institute of Technology, MS 320-47, Pasadena, CA 91225, USA; kym@caltech.edu \\ ${ }^{5}$ UC Berkeley, Astronomy Department, 601 Campbell Hall, Berkeley, CA 94720-3411, USA; saw@ astro.berkeley.edu \\ 614090 Robler Road, Sherman Oaks, CA 91423, USA \\ Received 2009 November 6; accepted 2010 May 24; published 2010 July 7
}

\begin{abstract}
Deep Keck/NIRC2 $H K^{\prime} L^{\prime}$ observations of the Arches cluster near the Galactic center reveal a significant population of near-infrared excess sources. We combine the $L^{\prime}$-band excess observations with $K^{\prime}$-band proper motions, which allow us to confirm cluster membership of excess sources in a starburst cluster for the first time. The robust removal of field contamination provides a reliable disk fraction down to our completeness limit of $H=19$ mag, or $\sim 5 M_{\odot}$ at the distance of the Arches. Of the 24 identified sources with $K^{\prime}-L^{\prime}>2.0 \mathrm{mag}, 21$ have reliable proper motion measurements, all of which are proper motion members of the Arches cluster. VLT/SINFONI $K^{\prime}$-band spectroscopy of 3 excess sources reveals strong $\mathrm{CO}$ bandhead emission, which we interpret as the signature of dense circumstellar disks. The detection of strong disk emission from the Arches stars is surprising in view of the high mass of the B-type main sequence host stars of the disks and the intense starburst environment. We find a disk fraction of $6 \% \pm 2 \%$ among B-type stars in the Arches cluster. A radial increase in the disk fraction from $3 \%$ to $10 \%$ suggests rapid disk destruction in the immediate vicinity of numerous O-type stars in the cluster core. A comparison between the Arches and other high- and low-mass star-forming regions provides strong indication that disk depletion is significantly more rapid in compact starburst clusters than in moderate star-forming environments.
\end{abstract}

Key words: circumstellar matter - Galaxy: center - open clusters and associations: individual (Arches) techniques: high angular resolution

Online-only material: color figures, machine-readable table

\section{INTRODUCTION}

\subsection{The Arches Starburst Cluster}

The Arches cluster is a dense, massive, young stellar cluster located in the central molecular zone near the center of the Galaxy (Cotera et al. 1996; Figer et al. 1999, 2002; Stolte et al. 2002, 2005, 2002, 2008; Kim et al. 2006; Espinoza et al. 2009). With a total stellar mass of $2 \times 10^{4} M_{\odot}$ (Espinoza et al. 2009), it is considered one of the rare starburst clusters in the Milky Way. The young age of only $2.5 \pm 0.5 \mathrm{Myr}$ (Najarro et al. 2004) places it at an evolutionary state comparable to nearby star-forming regions. Nearby star-forming regions at these young ages, as observed, for example, in the Orion complex, display a significant fraction of circumstellar disks remaining from the star formation process (Haisch et al. 2001; Hernández et al. 2007, and references therein). As a starburst cluster, however, the Arches hosts 125 O-type stars (Figer et al. 2002; Stolte et al. 2005), some of which have already evolved to the earliest Wolf-Rayet (W-R) phases (Martins et al. 2008). For comparison, the Orion Nebula Cluster (ONC) hosts only 2-4 Otype stars (Hillenbrand 1997). Most of our knowledge of the first few million years of stellar evolution and disk depletion stems from nearby star-forming regions of significantly lower density and stellar mass, while the effects of the starburst environment on circumstellar material and the disk lifetime have rarely been probed.

\footnotetext{
* Based on observations made with the Keck II telescope on Mauna Kea, Hawai'i, and with ESO Telescopes at the Paranal Observatory under program ID 60.A-9026.
}

Increasing evidence for remnant accretion disks around young, massive OB stars is deduced from $K$-band spectroscopy in very young star-forming regions (e.g., M17: Hanson et al. 1997, Hoffmeister et al. 2006; NGC 3576: Blum et al. 2004, Figuerêdo et al. 2005) and UCHII regions (e.g., Bik et al. 2005, 2006, and references therein). The large fraction of near-infrared excess objects and the fact that early O-type stars are on the main sequence suggest very young ages of less than 1-2 Myr for these environments. Observing disk fractions at later evolutionary stages is complicated by the steeply decreasing disk fraction as clusters age (Haisch et al. 2001), and the decreasing brightness of the increasingly depleted disks themselves. The Arches cluster, at an age of $2.5 \pm 0.5 \mathrm{Myr}$ (Najarro et al. 2004), fills one of the rare gaps in cluster evolution. Not only does it provide an estimate of the disk lifetime in its substantial B-star population, it additionally connects disk survival with the dense environment of a starburst cluster.

\subsection{Circumstellar Disks in Young Star Clusters}

In young star-forming regions with ages less than $10 \mathrm{Myr}$, $L$-band excess provides one of the most efficient tools to detect circumstellar disks (Haisch et al. 2001; see also Hillenbrand et al. 1992; Lada et al. 2000). As the illumination of the dense, inner disk rim by the central star generates $3.8 \mu \mathrm{m}$ emission from hot dust (Natta et al. 2001), the excess $L$-band flux indicates dense disks surviving from the star formation process. The hostile starburst cluster environment can accelerate disk destruction, to the extent that one might not expect to find any remaining gaseous disks in a cluster like the Arches. The finding of disks provides crucial clues for our understanding of 
disk survival and the likelihood of planet formation in dense cluster environments.

A linear decrease is observed in the disk fraction as a function of increasing cluster age in nearby star-forming regions (Haisch et al. 2001). This decrease in near-infrared excess emission can be understood as the depletion of dust in the inner, hot disk rim due to photoevaporation and grain growth (e.g., Takeuchi et al. 2005). The lifetimes of young disks, as derived from nearinfrared excess fractions, indicate that most disks are depleted within $10 \mathrm{Myr}$, with shorter disk lifetimes for higher-mass stars. In a pioneering study of 47 Herbig Ae/Be stars, Hillenbrand et al. (1992) found a more rapid depletion of disks around B-type stars with masses of $\sim 3-30 M_{\odot}$ than in A stars in the $1-3 M_{\odot}$ mass range. On average, the disks around high-mass stars do not survive for more than $1 \mathrm{Myr}$, with a characteristic disk age of only a few $10^{5} \mathrm{yr}$ (Alonso-Albi et al. 2009).

According to disk depletion simulations, the timescale for disk survival is expected to depend on the mass of the central star and on the ambient UV radiation field, and thus on the cluster environment. However, disk photoevaporation models do not arrive at a consistent conclusion for disk survival times around high-mass stars. Models of disks around high-mass $\mathrm{O}$ - and early B-type stars in isolation suggest that while the outer disk is evaporated by the central star on timescales of less than $1 \mathrm{Myr}$, the inner, dense disks with radii of $<10 \mathrm{AU}$ can survive for several Myr in late O-type stars, and more than $20 \mathrm{Myr}$ in early B-type stars (Richling \& Yorke 1997; Hollenbach et al. 2000; Scally \& Clarke 2001; see also Bik et al. 2006). Photoevaporation models by Takeuchi et al. (2005), on the other hand, predict that the dense, inner disk around a Herbig $\mathrm{Ae} / \mathrm{Be}$ star will be depleted out to a radius of $40 \mathrm{AU}$ within $3 \mathrm{Myr}$. In a starburst cluster, the numerous $\mathrm{O}$ - and B-type stars additionally create a strong EUV/FUV radiation field that accelerates disk evaporation. Simulations by Armitage (2000) suggest that a disk with a dust mass of $M_{d}=0.04 M_{\odot}$ can survive for up to $1 \mathrm{Myr}$ in clusters with $10^{4}$ stars at a distance of $1 \mathrm{pc}$ from the dense core, while the same disk will be evaporated in less than $10^{5} \mathrm{yr}$ if located at $0.1-0.3 \mathrm{pc}$ from the massive stars in the cluster center. Similar conclusions are reached by Fatuzzo \& Adams (2008), who simulate the influence of the radiation field on circumstellar disks in a massive star cluster with respect to planetary system formation. Tidal forces during dynamical interactions between cluster members might additionally truncate circumstellar disks (Olczak et al. 2008; Pfalzner et al. 2006; Cesaroni et al. 2007; Scally \& Clarke 2001; Boffin et al. 1998), and enhance the exposure of the inner disks to ambient UV radiation, thereby accelerating dust depletion.

In the Arches, NGC 3603, and similarly dense starburst clusters, the situation may be more extreme than in nearby starforming regions, as the numerous O-type stars segregate toward the cluster core, where the combined effect of their radiation fields acts on circumstellar material around lower-mass cluster members as well. The radiation environment in starburst clusters therefore has important consequences for the survival timescale of disks, both in the Milky Way and in extragalactic systems, and thus for the potential of planetary system formation in dense star-forming environments. In particular, the disk fraction is predicted to be a function of the stellar density in addition to the cluster age. A lower disk fraction in high-density environments might influence the accretion timescale and final stellar masses, and hence the initial stellar mass function. Observationally, the effect of the ambient stellar density can be evidenced as a depletion of circumstellar disks toward the dense cluster core, where the most massive stars reside. A decrease in the disk fraction with decreasing cluster radius is observed in the young starburst cluster at the center of the giant $\mathrm{H}$ II region NGC 3603 (Harayama et al. 2008; Stolte et al. 2004). As a consequence of limited spatial resolution and sensitivity in $L$-band observations, the high foreground extinction and large distances to Milky Way starburst clusters have so far hampered a systematic study of disk survival in the starburst environment. Thus far, NGC 3603 YC is the only starburst cluster where the disk fraction has been determined.

Fortunately, our study of the Arches cluster has provided an opportunity to progress in this domain. We have obtained $H K^{\prime} L^{\prime}$ observations of the Arches cluster with the Keck II laser guide star assisted adaptive optics system (LGS-AO) during our proper motion campaign to study the kinematics, extinction and stellar mass distribution of the cluster core. In the central $r<0.5 \mathrm{pc}$ of the cluster core, we detect 24 sources with substantial $3.8 \mu \mathrm{m}$ excesses of $\Delta\left(K^{\prime}-L^{\prime}\right) \geqslant 0.7 \mathrm{mag}$ as compared to the cluster main sequence population. VLT/ SINFONI $K$-band spectroscopy of three of these excess sources reveals $\mathrm{CO}$ emission, indicative of disk emission. The high contamination of field stars in the inner Galaxy particularly influences the fraction of main sequence sources at the faint end of the cluster main sequence population. The combination of a complete proper motion member sample among both the disk-bearing $L$-band excess sources and main sequence stars allows us to obtain the first measurement of the disk fraction in the Arches cluster. With the aim of assessing the prediction of an environment-dependent disk lifetime, we determine the disk fraction as a function of radius and discuss the prospects for disk survival around high-mass stars in a dense cluster environment.

The observations of the Arches cluster are presented in Section 2. Results are presented in Section 3, where the $K^{\prime}-L^{\prime}$ excess sample is defined in Section 3.1, $K$-band spectroscopy of selected sources is analyzed in Section 3.2, and the disk fraction of the Arches is derived in Section 3.3. The results are discussed in Section 4, including a comparison to similarly young star-forming regions (Section 4.2), and our results are summarized in Section 5.

\section{OBSERVATIONS}

\subsection{Keck/NIRC2 H $K^{\prime} L^{\prime}$ Imaging}

\subsubsection{Data Reduction and PSF Extraction}

Five fields in the Arches cluster were observed in $H K^{\prime} L^{\prime}$ with the Keck II NIRC2 camera (PI: K. Matthews) with the LGS-AO (Wizinowich et al. 2006; van Dam et al. 2006) between 2006 May and 2008 July. The field and tip-tilt star positions, distance from the brightest source in the cluster core and to the tiptilt source are provided in Table 1 . The central wavelengths and bandwidths for these NIRC2 filters are $\lambda_{H}=1.633 \mu \mathrm{m}$, $\delta \lambda_{H}=0.296 \mu \mathrm{m}, \lambda_{K^{\prime}}=2.124 \mu \mathrm{m}, \delta \lambda_{K^{\prime}}=0.351 \mu \mathrm{m}$, $\lambda_{L^{\prime}}=3.776 \mu \mathrm{m}$, and $\delta \lambda_{L^{\prime}}=0.700 \mu \mathrm{m}$. The narrow camera setting with a pixel scale of 0.00996 pixel $^{-1}$ delivered a field of view (FOV) of $10^{\prime \prime} \times 10^{\prime \prime}$ (Ghez et al. 2008). The LGS-AO system provided an $R=10 \mathrm{mag}$ wavefront reference source centered in the FOV. Two fainter natural guide stars with $R=15.3 \mathrm{mag}$ were used as tip-tilt reference sources, located $10^{\prime \prime}-15^{\prime \prime}$ from the center of each field. The AO performance was strongly dependent on the observing wavelength and on nightly atmospheric conditions. The average Strehl ratios were 


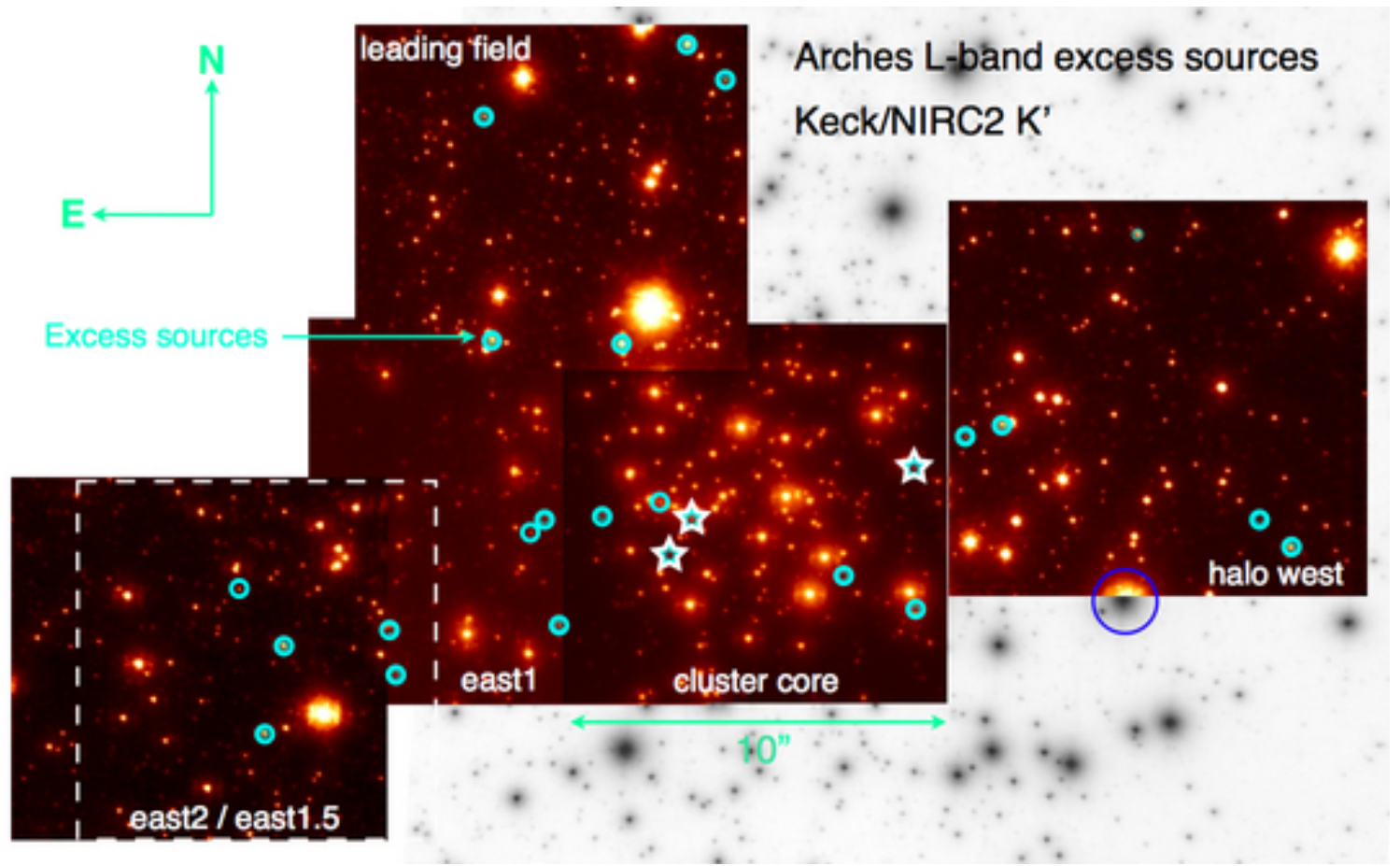

Figure 1. Arches Keck/NIRC2 $K^{\prime}$ mosaic image, with north up and east to the left. The tip-tilt reference star is indicated by the large circle, and $L$-band excess sources are encircled in light blue. The cluster core is visible as the area of high stellar density in the center of the image, fields east 1 and east 2 are offset to the east and southeast, the leading field is oriented along the proper motion axis to the north, and the halo field is located to the west and off the motion axis. The intermediate field east 1.5 is marked with a dashed box. Proper motion coverage is provided in the total area of the underlying gray-scale VLT-NAOS/CONICA image and the field east1.5. The three excess sources with SINFONI spectra are labeled with star symbols.

(A color version of this figure is available in the online journal.)

Table 1

Arches Cluster Fields Observed with Keck/NIRC2

\begin{tabular}{lccrrrr}
\hline \hline Field & R.A. & Decl. & $\delta$ R.A. $^{\text {a }}$ & $\delta$ Decl. $^{\text {a }}$ & Tip-tilt Star $^{\text {b }}$ & Distance to TTS $^{\text {C }}$ \\
Core & $17: 45: 50.540$ & $-28: 49: 20.07$ & 1.681 & -2.190 & 425 & 10.369 \\
East1 & $17: 45: 51.060$ & $-28: 49: 20.07$ & 8.620 & -2.190 & 425 & 17.200 \\
East1.5 & $17: 45: 51.600$ & $-28: 49: 24.40$ & 15.826 & 2.140 & 401 & 18.149 \\
East2 & $17: 45: 51.740$ & $-28: 49: 24.28$ & 17.694 & 2.020 & 401 & 16.352 \\
Lead & $17: 45: 50.960$ & $-28: 49: 11.72$ & 7.286 & -10.540 & 425 & 18.998 \\
Halo & $17: 45: 49.720$ & $-28: 49: 16.87$ & -9.261 & -5.390 & 425 & 5.603 \\
\hline
\end{tabular}

Notes.

${ }^{a}$ Positional offsets in right ascension and declination are given in arcseconds, relative to the brightest source in the cluster core (see Figure 1), R.A. 17:45:50.42, decl. -28:49:22.3. The cluster center is located at R.A. 17:45:50.54, decl. $-28: 49: 19.8$.

$\mathrm{b}$ Tip-tilt star identification numbers are given as extension from GSC2.2:S222122222, located at 425: R.A. = 17:45:49:78, 17:45:22.3, 401: 17:45:52.93, $-28: 49: 28.13$.

${ }^{c}$ The intermittent field east1.5 mostly overlaps with field east2, as well as a small section of field east1, to provide proper motion membership in the area not covered by the NACO field.

0.15 in $H, 0.27$ in $K^{\prime}$, and 0.64 in $L^{\prime}$. A $K^{\prime}$ mosaic of the five $H K^{\prime} L^{\prime}$ fields is illustrated in Figure 1, and field positions and tip-tilt star offsets are provided in Table 1. A summary of the observations, including the observational details, the number of frames entering each final combined image, as well as the resulting Strehl ratios and spatial resolutions, can be found in Table 2.

The images were reduced with our NIRC2 python/pyraf pipeline ( $\mathrm{Lu}$ et al. 2009), which includes dark and flat-field correction, sky subtraction, the removal of NIRC2 bad pixels and cosmic rays, and distortion correction (Thompson et al. 2001) prior to image combination. Ten separate sky frames were observed in each filter at the end of each $3 \mathrm{hr}$ Arches observing block. From these open-loop, star-free exposures, a median sky frame was created for each filter, which was scaled to the median background value of each individual exposure prior to subtraction. In $L^{\prime}$, a special procedure was employed to additionally account for structured thermal background caused by the Keck II field rotator mirror. Residual background structure was minimized by observing sky frames at the same rotator mirror angle as the science observations. Skies were taken with rotator angle increments of $2^{\circ}$, and median skies were averaged from three adjacent rotator angle positions. We also note that the best performance is achieved in $L^{\prime}$ when sky frames are taken close in time to the observations. When sky frames were observed within $1 \mathrm{hr}$ of our $L^{\prime}$ targets, the photometric sensitivity in the reduced images was typically 0.5 mag deeper than on the images observed earlier during the same night. Strehl ratios and full width at half-maximum (FWHM) values of the point-spread function (PSF) were derived for each individual image during 
Table 2

Log of Keck/NIRC2 Imaging and VLT/SINFONI Spectroscopy Arches Observations

\begin{tabular}{|c|c|c|c|c|c|c|c|c|c|c|c|}
\hline Date & Field & Filter & $t_{\exp }(\mathrm{s})$ & Co-adds & $N_{\text {obs }}$ & $N_{\text {used }}^{\mathrm{a}}$ & $t_{\text {int }}(\mathrm{s})$ & FWHM (mas) & Strehl $^{\mathrm{b}}$ & PSF Size $^{c}$ (pixel) & LF Peak $^{\mathrm{d}}$ \\
\hline \multicolumn{12}{|c|}{ Keck/NIRC2 Imaging } \\
\hline \multirow[t]{4}{*}{2006 May 21} & Core & $H$ & 10 & 6 & 15 & 15 & 900 & 63.2 & 0.17 & 100 & 19.0 \\
\hline & Core & $L^{\prime}$ & 0.5 & 60 & 15 & 15 & 450 & 79.4 & 0.64 & 47 & 14.0 \\
\hline & East1 & $L^{\prime}$ & 0.5 & 60 & 16 & 16 & 480 & 81.3 & 0.58 & 40 & 13.5 \\
\hline & $\mathrm{GC}^{\mathrm{e}}$ & $L^{\prime}$ & 0.5 & 60 & 19 & 19 & 570 & 81.4 & 0.38 & 100 & 12.0 \\
\hline \multirow[t]{6}{*}{2006 Jul 18} & Core & $K^{\prime}$ & 3 & 10 & 52 & 36 & 1080 & 56.0 & 0.22 & 100 & 19.5 \\
\hline & East1 & $H$ & 10 & 6 & 16 & 14 & 840 & 67.4 & 0.10 & 80 & 20.5 \\
\hline & East1 & $K^{\prime}$ & 3 & 10 & 15 & 10 & 300 & 65.0 & 0.15 & 90 & 18.5 \\
\hline & East2 & $H$ & 20 & 3 & 15 & 13 & 780 & 71.3 & 0.08 & 70 & 20.5 \\
\hline & East2 & $K^{\prime}$ & 3 & 10 & 15 & 15 & 450 & 59.3 & 0.22 & 75 & 19.5 \\
\hline & East2 & $L^{\prime}$ & 0.5 & 60 & 17 & 17 & 510 & 79.2 & 0.69 & 40 & 14.5 \\
\hline \multirow[t]{2}{*}{2007 Jun 21} & Leading & $K^{\prime}$ & 3 & 10 & 42 & 40 & 1200 & 56.0 & 0.38 & 80 & 20.5 \\
\hline & Halo west & $K^{\prime}$ & 3 & 10 & 28 & 26 & 780 & 53.7 & 0.38 & 80 & 19.5 \\
\hline \multirow[t]{5}{*}{2008 Jul 1} & Leading & $H$ & 10 & 3 & 29 & 29 & 870 & 58.7 & 0.20 & 100 & 20.5 \\
\hline & Leading & $L^{\prime}$ & 0.5 & 60 & 53 & 53 & 1590 & 80.8 & 0.55 & 80 & 15.5 \\
\hline & Halo west & $H$ & 10 & 3 & 23 & 23 & 690 & 50.5 & 0.23 & 100 & 20.5 \\
\hline & Halo west & $L^{\prime}$ & 0.5 & 60 & 39 & 39 & 1170 & 78.8 & 0.76 & 80 & 14.5 \\
\hline & East1.5 & $K^{\prime}$ & 3 & 10 & 22 & 21 & 630 & 68.0 & 0.29 & 100 & 19.5 \\
\hline \multicolumn{12}{|c|}{ VLT/SINFONI Integral-field Spectroscopy } \\
\hline 2006 May-Jul & Core & $H K$ & 300 & 24 & $\cdots$ & 7200 & $\cdots$ & 135 & $\ldots$ & $\ldots$ & $\cdots$ \\
\hline
\end{tabular}

Notes.

${ }^{\text {a }}$ FWHM and Strehl values are estimated from the averaged PSF image derived for each frame.

$\mathrm{b}$ The peak of the luminosity function indicates the completeness in each filter on each field.

c The PSF size is the diameter of the PSF extracted with starfinder and used to perform PSF fitting across each field (see Section 2.1).

${ }^{\mathrm{d}}$ Frames for the combined image were selected on the basis of the FWHM and the AO performance. For observing sequences with less than 20 frames, all frames with $\mathrm{AO}$ correction are included in the final image, while for sequences with more than 20 frames selection based on the FWHM in each image was possible (see Section 2 for details).

e GC refers to the Galactic center data set used for $L^{\prime}$ calibration.

the pipeline reduction. In observing sequences with less than 20 frames, all frames with closed-loop AO correction were combined into the final image. In observing sequences with more than 20 frames, frame selection to enhance the spatial resolution was possible without compromising photometric depth. In these sequences, only frames with $\mathrm{FWHM}<1.25 \times \mathrm{FWHM}_{\text {min }}$ were included in the final image, where $\mathrm{FWHM}_{\text {min }}$ was the minimum FWHM achieved in the sequence for each field and filter. This rejection criterion typically removed between $10 \%$ of the images under stable atmospheric conditions and $30 \%$ of the images in the case of variable seeing and $\mathrm{AO}$ performance prior to image combination. When frame selection was applied, the selected images were weighted by the Strehl ratio to enhance the resolution on the combined image. After the standard reduction procedures, the drizzle algorithm was used to combine the images to preserve the original resolution and to achieve the maximum sensitivity on the co-added image (Fruchter \& Hook 2002).

Relative photometry was extracted using the starfinder algorithm (Diolaiti et al. 2000), which is designed to fit an empirical PSF to crowded stellar fields. The code has the advantage of creating an empirical two-dimensional PSF by averaging several isolated sources, such that the extracted PSF is independent of fitted analytical functions. This is particularly crucial, as the Keck AO PSF cannot be characterized by azimuthally symmetric functions. The employed version of the code is limited to non-spatially varying PSFs. The radial extent of the PSF was set for each field and filter by the radius where the PSF structure was dominated by background noise, and is listed in Table 2 . In the cluster core, $\approx 10$ sufficiently bright and isolated sources could be found to define the average PSF. In the east fields (see
Figure 1), the rapidly decreasing density of bright stars limited the PSF template selection to 5-7 sources on field east1, and $3-5$ sources on east 2 . The final PSF for each field was created iteratively after selection and subtraction of neighboring sources from each image. Three iterations were performed before the final PSF was extracted. The average empirical PSF then served as the template to fit simultaneous photometry for all sources in each frame.

In addition to the deep, combined images, the reduced frames were divided into three qualitatively comparable subsets and combined into three auxiliary images to estimate the photometric and astrometric uncertainties. The auxiliary frames are composed from the list of all selected images sorted by Strehl ratio. From this quality-sorted list, every third frame enters auxiliary frames 1,2 , and 3 , respectively, to ensure the same quality in all three auxiliary frames. The random dither pattern with three images per pointing position and small dither offsets additionally ensures that image distortions are sampled in the same way in all three auxiliary frames. Starfinder positions and photometry were derived for point sources on the auxiliary images in the same way as for the deep data sets. As a first pass at rejecting faint, spurious detections caused by the varying halo structure of the PSF or fluctuations in the background, sources in $H$ and $K^{\prime}$ were required to be detected in at least one auxiliary frame in addition to the deep image in order to be included in the final photometry table. In addition, the auxiliary frames were used to estimate the photometric and astrometric uncertainties. In $H$ and $K^{\prime}$, the uncertainties are dominated by crowding effects. The photometry of stars near bright sources is sensitive to the Strehl ratio of each image, and hence their individual measurements on the three auxiliary 


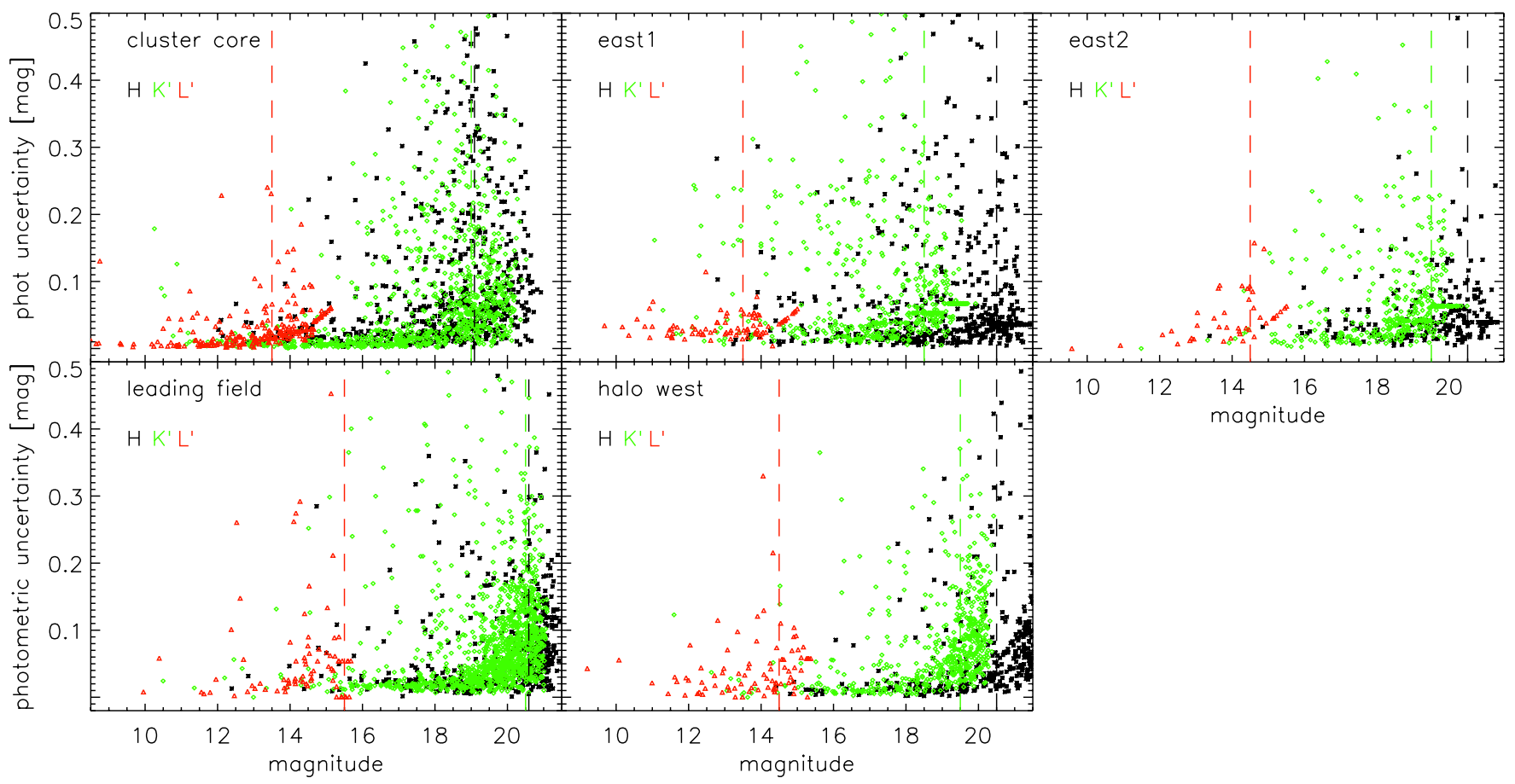

Figure 2. Photometric uncertainties for each field (black: $H$, green: $K^{\prime}$, red: $L^{\prime}$ ). The dashed lines indicate the observed peaks of the luminosity functions (LF). In the cluster core and leading field, the $H$ and $K$ LF peak is identical at 19 mag and $20.5 \mathrm{mag}$, respectively.

(A color version of this figure is available in the online journal.)

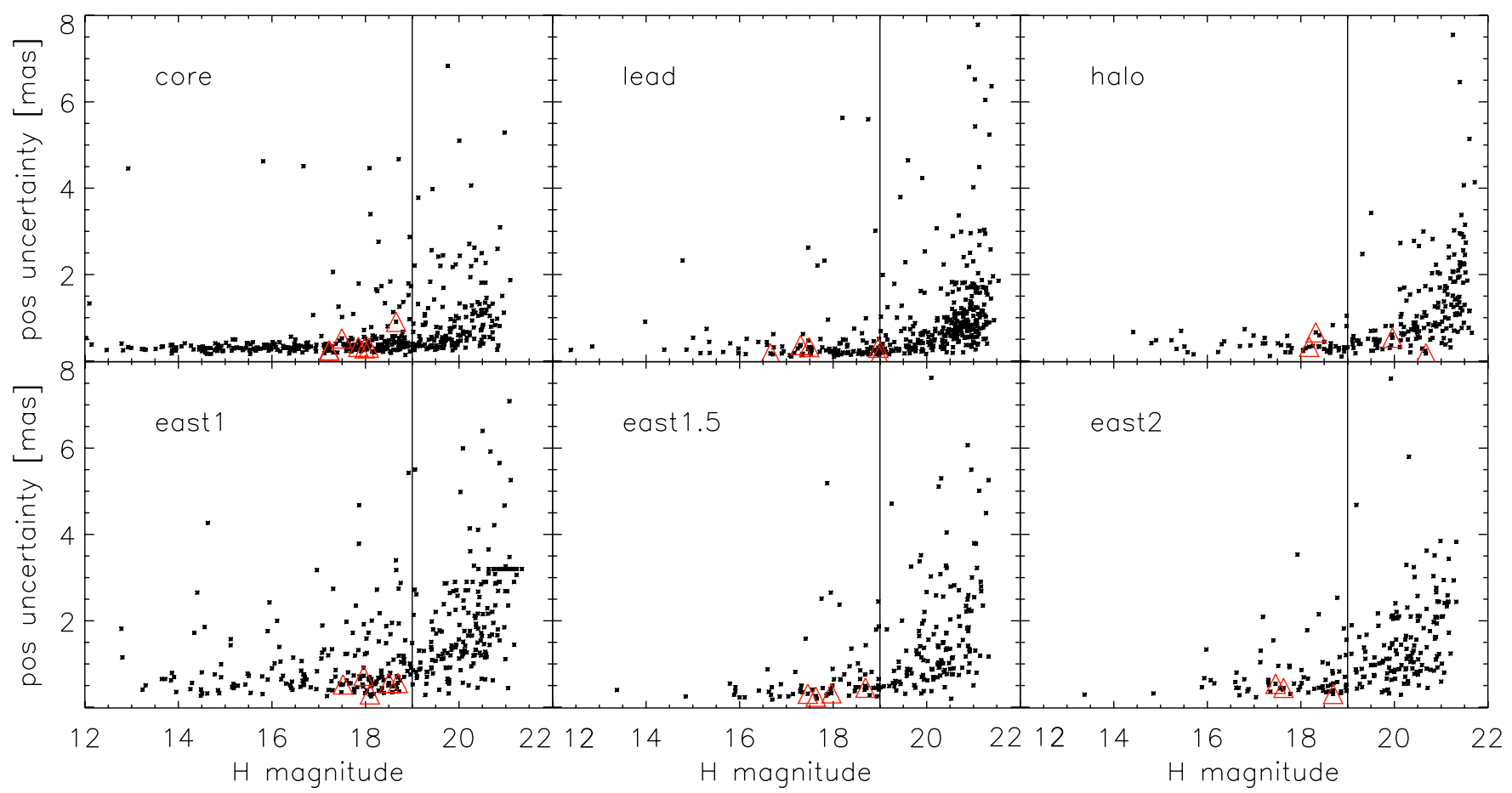

Figure 3. Positional uncertainties for each field measured from NIRC2 $K^{\prime}$ observations. The solid lines mark the imposed completeness limits at $H=19$ mag $L^{\prime}$-band excess sources are shown as triangles (red).

(A color version of this figure is available in the online journal.)

images vary more than for isolated sources. This crowding effect is not evident in the photometric uncertainties delivered during PSF fitting by starfinder, but is well reproduced in the rms uncertainties from repeated measurements on the auxiliary images. The astrometric and photometric uncertainties, shown in Figures 2 and 3, are derived from the standard deviation (rms) of the positions and fluxes on the auxiliary images and the main image, and hence from the repeatability of each measurement. In $L^{\prime}$, residual thermal background emission caused the sensitivity of the auxiliary images to be significantly lower than the sensitivity of the deep image, such that $L^{\prime}$ uncertainties are most affected by spatial sensitivity variations and not by crowding. The limited photometric sensitivity of the auxiliary frames restricted the number of $L^{\prime}$ sources matched with the photometry 
Table 3

Residual Zero-point Uncertainties

\begin{tabular}{lccccccc}
\hline \hline Field & $\begin{array}{c}\sigma_{H} \\
(\mathrm{mag})\end{array}$ & $N_{H}^{\mathrm{a}}$ & $\begin{array}{c}\sigma_{K^{\prime}} \\
(\mathrm{mag})\end{array}$ & $N_{K^{\prime}}^{\mathrm{a}}$ & $\begin{array}{c}\sigma_{L^{\prime}} \\
(\mathrm{mag})\end{array}$ & $N_{L^{\prime}}^{\mathrm{a}}$ & $\begin{array}{c}\text { Calibration Field } \\
H K^{\prime} \text { NACO/NIRC2 core }\end{array}$ \\
\hline Core $^{\mathrm{b}}$ & 0.010 & 56 & 0.009 & 64 & 0.100 & $\ldots$ & $\begin{array}{c}L^{\prime} \text { Galactic center zero point } \\
\text { East1 }\end{array}$ \\
East1.5 & 0.012 & 100 & 0.012 & 72 & 0.100 & 31 & $H K^{\prime} L^{\prime}$ core \\
East2 & $\ldots .024$ & 17 & 0.023 & 103 & 0.101 & $K^{\prime}$ core \\
Lead & 0.019 & 29 & 0.017 & 29 & 0.107 & 7 & $H L^{\prime}$ core, $K^{\prime}$ east1.5 \\
Halo & 0.010 & 107 & 0.006 & 228 & 0.101 & 30 & $H K^{\prime} L^{\prime}$ core \\
\hline
\end{tabular}

Notes.

a Number of calibration sources available. In the core $L^{\prime}$ data, the Galactic center (GC) zero point was applied (see Appendix B).

b The core $H K^{\prime}$ zero point was derived from the NACO central region, 250 pixels $<x, y<750$ pixels (NIRC2) to minimize anisoplanatic and -kinetic effects. The core $L^{\prime}$ zero point was derived from the GC standard field, all adjacent fields were calibrated with respect to the core field. The standard deviation in photometric residuals after calibration is given as the $H K^{\prime}$ zero-point uncertainties. The $L^{\prime}$ uncertainty is the zero-point variation during the GC observing sequence in the core field, and the variation between calibration sources is taken into account for the surrounding fields.

Table 4

Photometry and Astrometry Source List of the Arches Cluster

\begin{tabular}{|c|c|c|c|c|c|c|c|c|c|c|c|c|c|c|c|c|}
\hline ID & Field & $\begin{array}{c}\delta \text { R.A. } \\
\left({ }^{\prime \prime}\right)\end{array}$ & $\begin{array}{c}\delta \text { Decl. } \\
\left({ }^{\prime \prime}\right)\end{array}$ & $\begin{array}{l}\sigma_{x} \\
\left({ }^{\prime \prime}\right)\end{array}$ & $\begin{array}{l}\sigma_{y} \\
\left({ }^{\prime \prime}\right)\end{array}$ & $\begin{array}{c}H \\
(\mathrm{mag})\end{array}$ & $\begin{array}{c}\sigma_{H} \\
(\mathrm{mag})\end{array}$ & $\begin{array}{c}K_{s} \\
(\mathrm{mag})\end{array}$ & $\begin{array}{c}\sigma_{K_{S}} \\
(\mathrm{mag})\end{array}$ & $\begin{array}{c}L^{\prime} \\
(\mathrm{mag})\end{array}$ & $\begin{array}{c}\sigma_{L^{\prime}} \\
(\mathrm{mag})\end{array}$ & $\begin{array}{c}\text { Baseline } \\
(\mathrm{yr})\end{array}$ & $\begin{array}{c}\mu_{\alpha \cos (\delta)} \\
\left(\mathrm{mas}_{\mathrm{yr}}^{-1}\right)\end{array}$ & $\begin{array}{c}\mu_{\delta} \\
\left(\operatorname{mas}_{\mathrm{yr}}{ }^{-1}\right)\end{array}$ & Member & Excess \\
\hline 1 & Core & 0.000 & 0.000 & 0.0010 & 0.0001 & 12.023 & 0.030 & 10.258 & 0.179 & 8.364 & 0.100 & 4.33 & -0.551 & 0.046 & 1 & 0 \\
\hline 2 & Core & 1.936 & 4.725 & 0.0013 & 0.0013 & 12.097 & 0.044 & 10.447 & 0.090 & 8.577 & 0.101 & 4.33 & -0.597 & -0.115 & 1 & 0 \\
\hline 3 & Core & 0.664 & 2.764 & 0.0004 & 0.0004 & 12.145 & 0.021 & 10.535 & 0.079 & 8.749 & 0.100 & 4.33 & -0.069 & 0.023 & 1 & 0 \\
\hline 4 & Core & -0.419 & 1.048 & 0.0003 & 0.0002 & 12.465 & 0.013 & 10.772 & 0.021 & 8.822 & 0.100 & 4.33 & 0.000 & 0.345 & 1 & 0 \\
\hline 5 & Core & 3.381 & -0.302 & 0.0003 & 0.0001 & 12.819 & 0.014 & 11.144 & 0.011 & 9.443 & 0.100 & 4.33 & -0.092 & -0.184 & 1 & 0 \\
\hline 6 & Core & 1.348 & 1.620 & 0.0005 & 0.0002 & 12.925 & 0.013 & 11.349 & 0.014 & 9.763 & 0.100 & 4.33 & -0.368 & -0.161 & 1 & 0 \\
\hline 7 & Core & -1.893 & 5.057 & 0.0003 & 0.0003 & 12.992 & 0.025 & 11.185 & 0.025 & 9.414 & 0.100 & 4.33 & 0.092 & 0.368 & 1 & 0 \\
\hline
\end{tabular}

(This table is available in its entirety in a machine-readable form in the online journal. A portion is shown here for guidance regarding its form and content.)

of the $H$ and $K^{\prime}$ images. With the goal of including the fainter $L^{\prime}$ sources detected only in the deep combined image in the final catalog, all $L^{\prime}$ starfinder detections were matched with the $H K^{\prime}$ photometric table. When sources were detected in the auxiliary frames, the $L^{\prime}$ photometric uncertainties were derived from the standard deviation of the repeated measurements. For faint sources not detected in the auxiliary frames, the starfinder signal-to-noise ratio $(\mathrm{S} / \mathrm{N})$ was used to estimate the photometric uncertainty. As PSF fitting does not account for background fluctuations, the starfinder $\mathrm{S} / \mathrm{N}$ still appears to underestimate the photometric uncertainty in some cases. A comparison of the rms photometric uncertainties derived from the auxiliary frames and the starfinder $\mathrm{S} / \mathrm{N}$ indicates that the starfinder $\mathrm{S} / \mathrm{N}$ may underestimate the true $L^{\prime}$ photometric uncertainty by up to a factor of 4 . These smaller uncertainties have a negligible effect on the final photometric uncertainty of each source, which is dominated by the uncertainty in the zero point (see Table 3 ). The final $H K^{\prime} L^{\prime}$ table thus contains as many sources as possible, while avoiding $L^{\prime}$ artefacts with the requirement that any $L^{\prime}$ source be detected in all three filters. This procedure resulted in a clean list of $380 H K^{\prime} L^{\prime}$ detections and 1033 additional $H K^{\prime}$ detections for the two-color analysis (Table 4).

All calibrated $H K^{\prime} L^{\prime}$ magnitudes and uncertainties are listed in the full version of Table 4, which can be downloaded from the online version of the journal.

\subsection{2. $H K^{\prime}$ Photometric Calibration}

The $H K^{\prime}$ photometry was calibrated with reference to the VLT/NAOS-CONICA (NACO) observations obtained in 2002 (Stolte et al. 2002, Stolte et al. 2005; see also Stolte et al.
2008), covering a $26^{\prime \prime} \times 27^{\prime \prime}$ FOV around the cluster center with a spatial resolution similar to the Keck/NIRC2 observations (Table 2). The absolute calibration zero point for the NACO photometry is provided by lower resolution ( 0 '.2) Hubble Space Telescope (HST)/NICMOS calibrations presented in Figer et al. (1999). Five available Two Micron All Sky Survey (2MASS) sources were used to check that the calibration with respect to the NICMOS filter system did not display systematic offsets with respect to the standard 2MASS $H K_{s}$ system. As in our previous work, no color terms were found (Figure 4). In order to verify that the use of a spatially invariant PSF did not alter the photometric performance across the field, photometric residuals were tested independently in the $x-, y$-, and radial directions. A possible weak zero-point variation of less than $\pm 0.1 \mathrm{mag}$, comparable to the standard deviation of $\sigma_{\Delta K}=0.10 \mathrm{mag}$ in $K^{\prime}$ and $\sigma_{\Delta H}=0.15 \mathrm{mag}$ in $H$ in a sample of 76 calibration sources, may be present over the maximum radial extent of 5".5 in the NIRC2 $H$ and $K^{\prime}$ data sets when compared to both NICMOS and NACO, but the linear fit indicates less than $1 \sigma$ significance. As the variance is within our formal zero-point uncertainties (Table 3), we apply a constant zero point across the field. With increasing distance from the field center, anisoplanatic effects are expected to affect the natural guide star observations with NACO most severely, while more modest effects are expected for the Keck LGS observations since the artificial guide star was pointed at the center of each $10^{\prime \prime} \times 10^{\prime \prime}$ FOV. This may explain the slight differences of $0.03-0.10$ between the zero points of the NIRC2-NIRC2 and the NIRC2-NACO calibrations. In order to achieve a uniform photometric calibration across the NIRC2 mosaic area, we therefore apply a two-step correction process. First, absolute photometric calibration is obtained for the central 

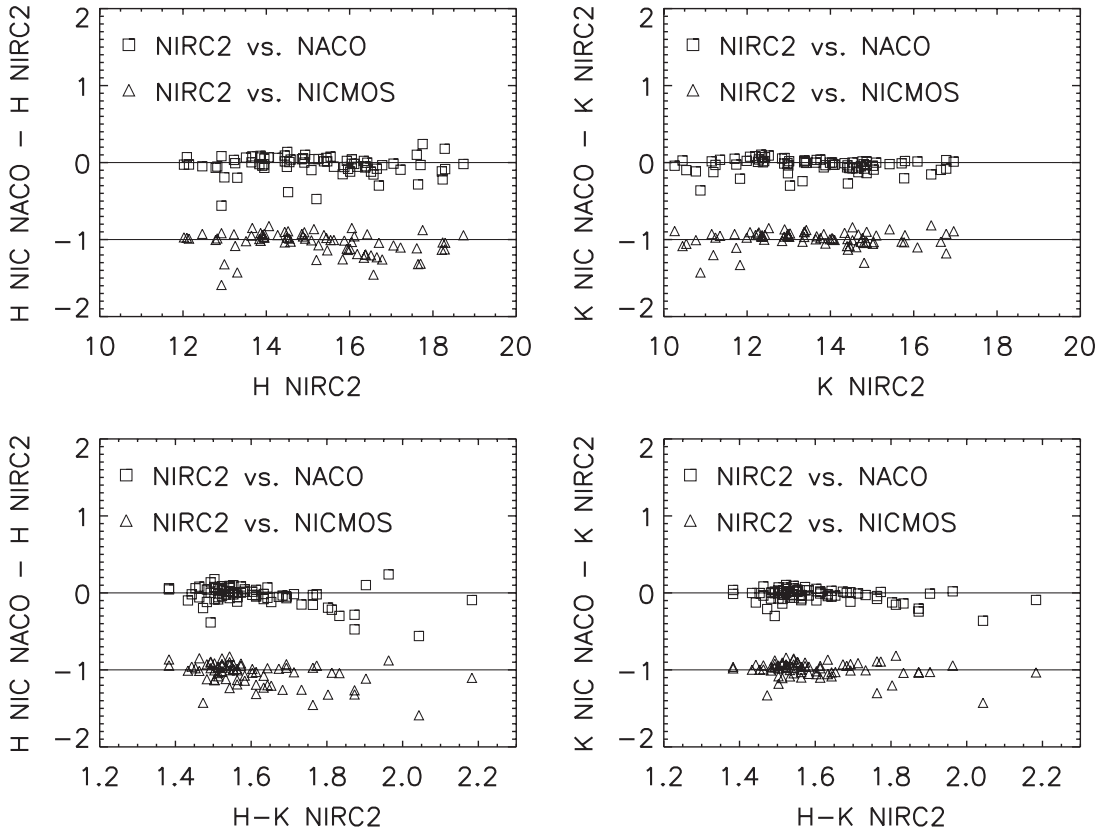

Figure 4. Color terms between NIRC $2 H$ and $K^{\prime}$ observations and NACO $H$ and $K_{s}$ as well as NICMOS $F 160 W$ and $F 205 W$ calibrations. The top panels show that there is no systematic trend between calibrated and reference magnitudes in the covered brightness regime of calibration sources. The residual magnitude differences vs. calibrated $H-K^{\prime}$ color (bottom panels) display no significant color terms. NIRC2 vs. NICMOS comparisons are shifted by -1 for clarity.

core region from the comparison with $\mathrm{NACO}$, and relative calibration of fields at larger distances from the guide star is then derived with respect to the cluster core. The core field is close to the guide star used both as the natural GS with NACO and as the tip-tilt source with NIRC2, such that anisoplanatic effects at increasing guide star distances are minimized (see Figure 1). For the core field, we use only the innermost $5^{\prime \prime}$ region within $250<x, y<750$ NIRC2 pixels to calibrate against NACO $H K_{s}$. The high stellar density in this area provides 56 sources with $\sigma_{K s}<0.1$ and $\sigma_{H}<0.15$ mag for the absolute calibration, and zero-point variations over this small area were confirmed to be negligible. The zero-point offsets of the adjacent fields, "east1" and "lead" were derived with respect to the calibrated core field, and "east 2 " and the intermediate proper motion field "east1.5" were calibrated against "east1" (see Table 3). Only sources with relative photometric uncertainties $\sigma_{H K^{\prime}}<0.1 \mathrm{mag}$, as derived from the auxiliary images (see above), were used as calibration sources. The halo field does not overlap with the core field, but its proximity to the guide star ensures that anisoplanatic effects are avoided. Hence, the halo field $H K^{\prime}$ photometry was calibrated against the NACO field. The average zero points after airmass correction were 25.219 in $H$ and 24.578 in $K^{\prime}$.

\subsubsection{L' Photometric Calibration}

In $L^{\prime}$, where no previous data exist, absolute calibration was achieved using $L^{\prime}$ observations of the central parsec in the Galactic center (GC), where numerous previous long-wavelength observations are available (see Appendix A, Table A.1, and for reference, see also Blum et al. 1996; Clénet et al. 2001, 2004). The central parsec was observed during the same night and at the same airmass as the Arches core field. The surrounding Arches fields were subsequently calibrated relative to the core field. The same observational setup was employed for both the Arches and the GC observations. Taking into account airmass variations, we first derived the zero point for the GC data by referencing against calibrated NGSAO observations reported in Appendix A. The final calibration was confirmed by matching with both the Blum and Clénet tables. This procedure ensures that the zero-point offset between the $L^{\prime}\left(\lambda_{c}=3.75 \mu \mathrm{m}\right)$, used by Clénet et al. (2001) and for our data, and the $L\left(\lambda_{c}=3.45 \mu \mathrm{m}\right)$ filter used in Blum et al. (1996) was accounted for. The difference between $L$ and $L^{\prime}$ is a constant offset in this case, as sources in the GC sample as well as in the Arches cluster have comparable line-of-sight extinctions (see Appendix B for details on the filter differences and the extinction law). ${ }^{7}$ The uncertainty in the zero point was estimated from variations of the zero points of individual images throughout the observation sequence. The derived GC $L^{\prime}$ zero point of $23.30 \pm 0.10$ mag was used to calculate magnitudes for the Arches core field after airmass correction. Possible temperature or atmospheric variations leading to a varying thermal or sky background during the $2 \mathrm{hr}$ of $L^{\prime}$ observations cause the remaining uncertainty of $0.10 \mathrm{mag}$ in the zero point. When the calibrated photometry was compared to the location of the reddening vector in the $H-K^{\prime}, K^{\prime}-L^{\prime}$ two-color diagram, the majority of sources was located below the reddening line. This is unexpected, as the cluster population is dominated by main sequence stars, and the field population is dominated by field giants. The intrinsic colors of main sequence stars are close to zero in the standard magnitude system, such that the reddening vector also passes through the origin in the two-color diagram, and main sequence stars behind substantial foreground extinction, such as the Arches members, are expected to scatter around this vector according to their photometric uncertainties. A shift of $\Delta L^{\prime}=+0.1 \mathrm{mag}$ allowed for both cluster stars and background sources in the Arches field to follow the extinction vector (see Section 3.1.1), as expected. Such a zero-point shift can be explained by the difference in the average extinction between the GC calibration sources, $\left\langle A_{V}\right\rangle=33.1 \mathrm{mag}$ and Arches cluster members, $\left\langle A_{V}\right\rangle=25.3 \mathrm{mag} . \Delta A_{V}=7.8 \mathrm{mag}$ corresponds to a difference of $\Delta L^{\prime}=0.12$ mag when calibrating $L^{\prime}$ with respect

\footnotetext{
7 Note that Viehmann et al. (2005) provided the most recent photometry in the central parsec, however, these data unfortunately display an extensive scatter in $K^{\prime}$ as well as $L^{\prime}$, such that they could not be used as calibration sources.
} 
to $L$ data (see Appendix B for details). This offset is, however, also consistent with the zero-point variation across the $2 \mathrm{hr} L^{\prime}$ GC observing sequence due to thermal background variations across the night. As the offset is within the zero-point uncertainties, it was applied to the absolute $L^{\prime}$ calibration.

All adjacent images were calibrated with respect to the core, and as in the case of $H K^{\prime}$, the zero-point uncertainties were calculated based on the standard deviations of the photometric residuals of the calibration sources after the zero point was applied. In the halo field, four $L^{\prime}$ acquisition exposures with total exposure times of $30 \mathrm{~s}$ each were obtained with one third of the acquisition images overlapping the core field, and two thirds overlapping the lower-density halo field. The acquisition exposures were combined to provide a calibration field for the halo field $L^{\prime}$ photometry. The zero point of the acquisition image was obtained with respect to the core, and the halo field was calibrated with respect to the acquisition image, consecutively. The reported systematic zero-point uncertainty in $L^{\prime}$ for this field is comprised of the standard deviations of the photometric residuals of the acquisition and halo fields.

\subsubsection{Zero-point Uncertainties}

Zero-point uncertainties are estimated from the standard deviation in the photometric residuals of calibration sources after the zero point was applied (Table 3), and are included as systematic uncertainties in all reported photometric uncertainties. Where several fields had to be used consecutively to obtain the absolute zero point, the standard deviations from each field were added in quadrature to obtain the final zero-point uncertainty. The zeropoint uncertainties in east 1 comprise the quadrature sum of the core NIRC2-NACO residuals (first row in Table 3) and east1 residuals $\left(\sigma_{H}=0.065, \sigma_{K^{\prime}}=0.063, \sigma_{L^{\prime}}=0.054\right)$. The intermediate field east 1.5 was only observed in $K^{\prime}$ for proper motion membership. The zero-point uncertainty contains the standard deviation from all consecutive calibrations (i.e., the zero-point uncertainties of $K^{\prime}$ core and $K^{\prime}$ east1), added in quadrature to the measured standard deviation in $K^{\prime}$ east $1.5\left(\sigma_{K^{\prime}}=0.086\right)$ after calibration. The east $2 H K^{\prime} L^{\prime}$ zero points were derived from field east 1.5 in $K^{\prime}$, and field east 1 in $H$ and $L^{\prime}$, and the uncertainties contain the uncertainty in the core, east 1 and east 1.5 calibration in addition to the residual standard deviation in east2 after calibration $\left(\sigma_{H}=0.088, \sigma_{K^{\prime}}=0.073, \sigma_{L^{\prime}}=0.030\right)$. The leading field $H K^{\prime} L^{\prime}$ zero points were derived from overlap with the core field, and the uncertainties include the quadrature sum of the core zero-point uncertainties and the standard deviation in the residuals of the leading field after calibration $\left(\sigma_{H}=0.086\right.$, $\sigma_{K^{\prime}}=0.079, \sigma_{L^{\prime}}=0.100$ ). The halo field $H K^{\prime}$ photometry was zero pointed against the NACO $H K$ catalog. The zero-point uncertainty is the residual standard deviation in the calibration sources after the zero point was applied. $L^{\prime}$ photometry on the halo field was calibrated in two steps with respect to an acquisition exposure with significant overlap to the core and halo fields. The $L^{\prime}$ zero-point uncertainty contains the uncertainties in the core and acquisition image calibrations in addition to the residual standard deviation in the halo field after the zero point was applied $\left(\sigma_{L^{\prime} \text { core }}=0.100, \sigma_{L^{\prime} \text { aqu }}=0.064, \sigma_{L^{\prime} \text { halo }}=0.054\right)$. Final zero-point uncertainties from this procedure are tabulated in Table 3.

\subsubsection{Photometric Uncertainties and Completeness Limits}

Figure 2 shows the relative photometric uncertainties in each field, color-coded by filter. As discussed above, the relative uncertainties were derived from the repeatability of each photometric measurement from the three auxiliary images in each field and filter. These uncertainties illustrate the filter dependence in the photometric accuracy and the uniformity between the different observations. The absolute uncertainty is dominated by the systematic zero-point uncertainty, which appears as a constant offset in these plots and is not included here, such that the relative measurement uncertainties can be directly compared. The dashed lines indicate the peak in the observed luminosity functions (LFs) as a proxy for the completeness limit. In the crowding-limited field of the Arches core, the non-detection of sources in high-density regions shifts the peak of the LF to brighter luminosities. While numerous stars can thus be detected at magnitudes fainter than the LF peak, the sample beyond this limiting magnitude will be incomplete. In field east 1 (middle panel), where only 10 frames with good AO correction were obtained, the $K^{\prime}$-band photometry is significantly shallower. Where sources were detected in the overlap regions between east1 and the cluster core or east2, sources were preferentially selected from the more complete core and east 2 photometric samples. The last column in Table 2 contains the peak of the LF in each field and filter, which we identify as the completeness limit. In the crowding-limited cluster core, the $H K^{\prime} L^{\prime}$ completeness limits are 19.0, 19.5, and 14.0, and in the remaining fields the average limits are 20.5, 19.5, and 14.5 in $H K^{\prime} L^{\prime}$, respectively. The total numbers of unique sources detected in all three filters in the core, east1, east2, leading, and halo-west fields are 173, 32, 39, 73 , and 63 , respectively. These $H K^{\prime} L^{\prime}$ samples are limited by $L^{\prime}$ completeness, and faint sources without $L^{\prime}$-band excess will not be included in this sample. For the derivation of the excess fraction, it is crucial to have a complete main sequence reference sample (see Section 3). Hence, we also define a sample of $H K^{\prime}$ detections down to the $H$-band completeness limit of 19.0 mag in the most crowding-limited cluster core. The $H K^{\prime}$ sample includes $261,63,75,108$, and 61 unique detections with $H<19.0$ mag in the core, east1, east2, leading, and halo-west fields, respectively. Note that these samples are not yet selected for cluster membership, but include the full number of detections in our fields.

\subsection{Proper Motion Membership}

Following the procedures detailed in Stolte et al. (2008), the $K^{\prime}$-band catalog was matched with VLT/NAOS-CONICA observations, providing a time baseline of $4.3 \mathrm{yr}$. As different fields were observed over several years with NIRC2 during different atmospheric conditions, the geometric transformation of each NIRC2 $K$-band image was derived with respect to the larger NACO image individually. The transformations are derived iteratively, with the first pass determined from bright, likely cluster members, and the second pass from a preliminary selection of member candidates of all magnitudes to improve spatial coverage and thus the quality of the transformation. This procedure ensures that variations due to $\mathrm{AO}$ performance and airmass differences are removed. Note that this also implies that internal cluster motions, which are expected to be below our NIRC2-NACO proper motion accuracies, are also minimized and cannot be detected with this method. A comparison of NIRC2-NIRC2 epochs leading to the determination of internal motions will be the topic of a forthcoming paper (W. Clarkson et al. 2010, in preparation). In addition to the core members determined in Stolte et al. (2008), we have included sources with membership information on field east 1 , as well as on the leading and halo-west fields, where overlap with 


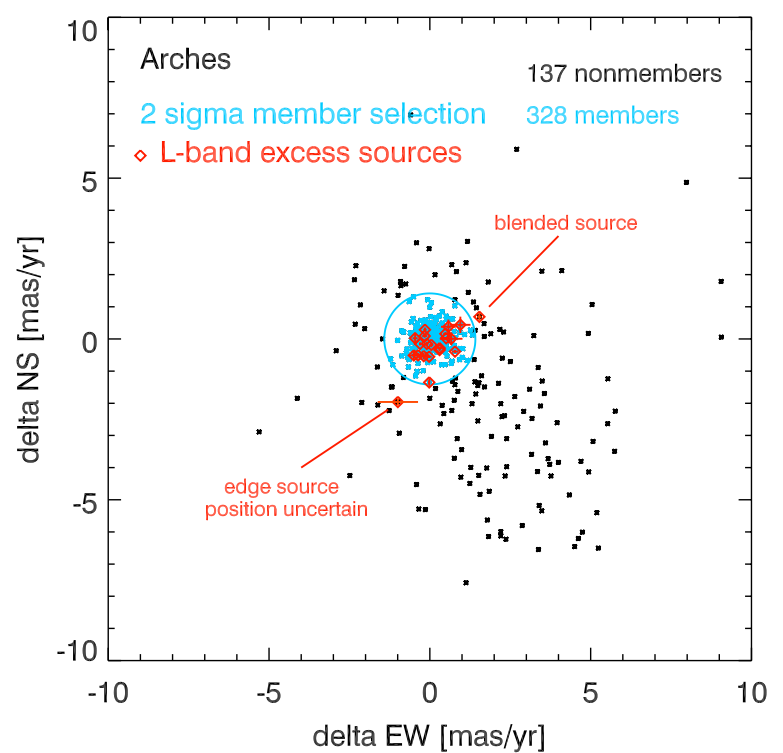

Figure 5. Proper motion membership selection of stars with $H<19$ mag. Cluster members are selected within $2 \sigma$ of the FWHM derived from a Gaussian fit to the motion distribution. Excess sources are marked as red diamonds Positional uncertainties for most excess sources are smaller than the symbol size. The large uncertainty of the one excess source below the proper motion selected cluster member sample (cyan) does not permit membership determination for this source. The second formal non-member in the excess sample is blended with a nearby star in the NACO image. Both sources are excluded from the member disk sample.

(A color version of this figure is available in the online journal.)

the NACO data set is available (see Figure 1). A total area of $442 \operatorname{arcsec}^{2}$ was observed in $H K^{\prime} L^{\prime}$, of which $312 \operatorname{arcsec}^{2}$ are also covered with NACO. Fields east1 and east 2 did not overlap entirely with our NACO observations. The intermediate field east1.5 covering the missing portion of the eastern part of the cluster was re-observed with Keck/NIRC2 in 2008 July to obtain second-epoch $K^{\prime}$ images (see Table 2), which increases the area with proper motion measurements to $392 \operatorname{arcsec}^{2}$. Hence, $90 \%$ of the $H K^{\prime} L^{\prime}$ coverage has proper motion information. The standard deviation in the proper motions of cluster members derived for fields east $1 /$ east 2 using east1.5 as proper motion reference over a two-year baseline with NIRC2 was comparable to the astrometric residuals in the four-year NACO-NIRC2 comparison, such that no distinction between the two data sets had to be made.

Only stars brighter than the core $H$-band incompleteness limit (peak of the LF), $H<19$ mag, were included in the fit, as these stars represent the main sequence sample in the analysis below. The membership selection of stars brighter than $H=19$ mag is illustrated in Figure 5. The Gaussian fit to the strong concentration of stellar motions in this sample yields a

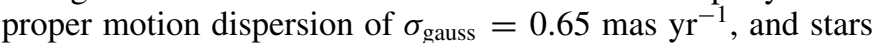
within $2 \sigma$ of the peak, or a proper motion of less than 1.30 mas $\mathrm{yr}^{-1}$ relative to the average motion of the Arches cluster, were considered cluster member candidates (hereafter "proper motion members"). In the following sections, members and nonmembers will be distinguished on the basis of this selection criterion. As a consequence of the different resolutions in the two data sets, the proper motion table is not complete in the cluster core. Faint sources or close neighbors resolved with the 53 mas resolution obtained in the NIRC2 $K^{\prime}$-band images were not always resolved with the 84 mas resolution obtained with NACO. This leads to 16 sources without proper motion information in the cluster core, one of which has $L^{\prime}$-band excess (see Section 3). The membership status of the 15 sources without $L^{\prime}$ excess is uncertain, and therefore these are not considered members. The detection of $L^{\prime}$-band excess suggests that this one sources belongs to the cluster, such that this source is included, albeit separately, in the discussion below.

This procedure provides membership information for 479 (84\%) sources of the $568 H K^{\prime}$ detections with $H<19$ mag, including 335 cluster members. The final $H K^{\prime} L^{\prime}$ catalog, which is limited by the $L^{\prime}$ detection threshold of $\sim 14$ mag, contains 362 detections with $H<19$ mag, of which 331 (91\%) have proper motion information and 235 are cluster member candidates within the $2 \sigma$ member selection criterion.

\subsection{VLT/SINFONI K-band Spectroscopy}

VLT/SINFONI integral-field spectroscopy was obtained in the core of the Arches cluster between 2006 May and 2006 July. The medium pixel scale with $50 \times 100$ mas pixel $^{-1}$ spaxels delivered an FOV of $3^{\prime \prime} \times 3^{\prime \prime}$, and the $H+K$ grism yielded a mean spectral resolution of 1750 . Two fields in the cluster core were observed, and the total integration time was $2 \mathrm{hr}$ per field split into four spectral cubes of 30 minutes each. Each cube consisted of six individual $300 \mathrm{~s}$ exposures obtained with an ABBA dither pattern alternating between science and sky frames pointed to a dark region near the cluster to account for variable sky levels in the near-infrared. The spectra were extracted from the pipelinereduced data delivered by ESO, which includes standard sky subtraction, flat fielding, masking of hot pixels, and wavelength distortion correction and calibration. The wavelength scale was rebinned to an equal wavelength spacing of $0.0005 \mu \mathrm{m} \mathrm{pixel}{ }^{-1}$, providing a nominal spectral resolution of 2000 at $2.2 \mu \mathrm{m}$ or $140 \mathrm{~km} \mathrm{~s}^{-1}$ per wavelength element. From telluric lines, a spectral resolution of 1900 is measured near $2.2 \mu \mathrm{m}$, or $160 \mathrm{~km} \mathrm{~s}^{-1}$ (FWHM). Telluric correction was achieved either with a G2V or a B3V calibrator observed during the same night. In the case of the $\mathrm{G}$ star, the rebinned, high-resolution solar spectrum available from KPNO was used to remove intrinsic stellar features, while for the B3V calibrator, the broad hydrogen lines were removed using template spectra from the $K$-band atlas of Hanson et al. (1996). In order to correct for flexures in the SINFONI instrument, the telluric spectra were crosscorrelated with bright stars in the science cubes, sub-pixel shifted and rebinned to the original wavelength scale. This procedure resulted in a clean removal of almost all telluric lines, except for one residual line at $2.355 \mu \mathrm{m}$, visible as a strong contaminant at the location of the third $\mathrm{CO}$ overtone in all the spectra. This line might originate locally near the telescope or inside the instrument, as telluric sky correction did not allow for its removal in either of our science data cubes. Three sources with $L^{\prime}$ excess (see Section 3.1.1) were located in two SINFONI fields. For these three $L^{\prime}$-band excess sources with $K=15.9,15.2$, and 15.5 , the spectra of three to four data cubes were added to provide the final, extracted spectrum of each source with S/N's of 40,65, and 60, respectively, in the $K$ continuum near the $\mathrm{CO}$ emission band heads.

\section{RESULTS}

\subsection{Photometric and Spectroscopic Evidence for Circumstellar Disks in the Arches Cluster}

\subsubsection{Identification of $L^{\prime}$-band Excess Sources}

Near-infrared excess at wavelengths longward of $3 \mu \mathrm{m}$ can be caused by circumstellar dust emission. At different stages 

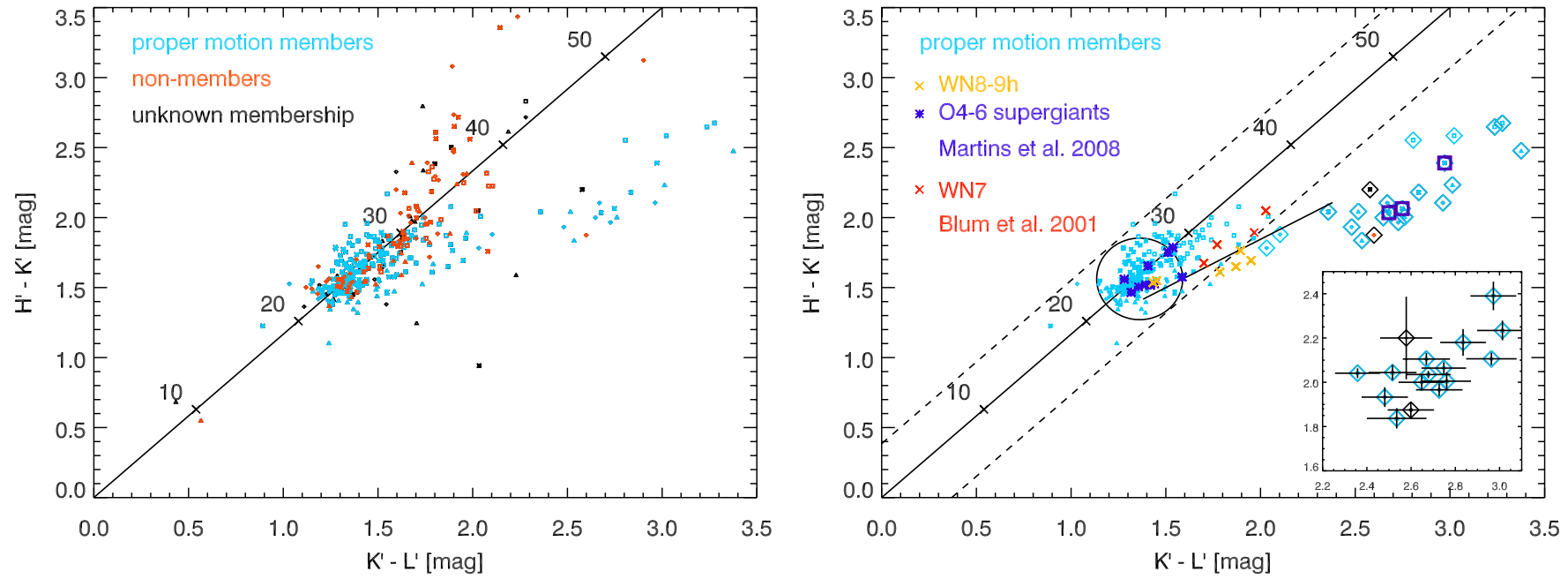

Figure 6. $H-K^{\prime}, K^{\prime}-L^{\prime}$ two-color diagram of sources in the Arches cluster fields. The solid line indicates the reddening path of an A0 star using the extinction law as measured toward the GC (Rieke \& Lebofsky 1985). Left: proper motion members (light blue) preferentially cluster below extinctions of $A_{V}<30$ mag, while non-members (red) scatter to extinction values as high as $A_{V} \approx 50 \mathrm{mag}$, as expected along the GC line of sight. Sources with $L^{\prime}$-band excess are located significantly to the right of the reddening vector. Right: $H-K^{\prime}, K^{\prime}-L^{\prime}$ diagram with Arches members. The two excess sources without membership information (black diamonds) are included for completeness. The ellipse marks the $2 \sigma$ standard deviation in $H-K^{\prime}$ and $K^{\prime}-L^{\prime}$ (note the different axis stretch in $H-K^{\prime}$ and $K^{\prime}-L^{\prime}$, causing the ellipse to appear as a circle), and the dashed lines are the tangents of the uncertainty ellipse parallel to the reddening vector. Main sequence cluster members with enhanced foreground extinction are expected to occupy the space between the dashed lines. Excess sources are selected redward of the lower dashed line if their photometric uncertainty indicates a significant offset from this line. The excess sources (diamonds) stand out at colors redder than $K^{\prime}-L^{\prime}=2.0 \mathrm{mag}$. The inset shows excess sources with photometric uncertainties. The classical T Tauri locus is included as a dash-dotted line for reference (CTTS; Meyer et al. 1997). Blue asterisks and yellow crosses denote young, high-mass stars with known spectral types (Martins et al. 2008). Red crosses are evolved bright clusters members $\left(K^{\prime}<11.5\right.$ mag) without spectral types. The bulk of the excess source population has substantially more excess than expected for young, lower-mass stars with disks (CTTS), or evolving hydrogen-rich Wolf-Rayet stars (yellow crosses) surrounded by dusty envelopes.

(A color version of this figure is available in the online journal.)

of stellar evolution, the emitting molecular material can be found in different geometries around the central star. In very young main sequence stars, dust emission is attributed to the existence of a circumstellar disk, assumed to be the remnant disk from the accretion phase of the star. A remnant disk is less likely for high-mass stars earlier than spectral type B3V, where native material is evaporated by UV radiation from the central star on timescales of 1 to a few Myr (Richling \& Yorke 1997; Hollenbach et al. 2000). At later stages of high-mass stellar evolution, in particular during the W-R phase, the winds of high-mass stars can produce massive, dusty envelopes, in which the stellar light is also reprocessed and emitted at nearto mid-infrared wavelengths (see Crowther 2007 for a review).

The $H-K^{\prime}, K^{\prime}-L^{\prime}$ two-color diagram (Figure 6) allows us to distinguish sources with enhanced foreground extinction from infrared excess sources. The solid line indicates the reddening path of an A0 star using the extinction law as measured toward the GC (Rieke \& Lebofsky 1985). In Figure 6 (left), cluster members are plotted in light blue, while non-members are displayed in red. Black dots indicate stars without membership information. The bulk of the Arches members follow the reddening vector, as expected from the range of extinctions of $22 \mathrm{mag}<A_{V}<30 \mathrm{mag}$ observed toward the cluster center (see also Stolte et al. 2002; Espinoza et al. 2009). Non-members are preferentially found at higher extinctions, but still concentrated around the reddening path. The proper motion member sample of Arches cluster stars is shown in the right panel of Figure 6. The efficiency of the membership selection is evidenced by the lack of sources above a foreground extinction of $A_{V} \sim 33 \mathrm{mag}$ along the reddening vector. A significant sample of extremely red cluster members with $K^{\prime}-L^{\prime}>2.3 \mathrm{mag}$ is found to the right of the reddening vector, clearly distinct from both the cluster main sequence and the field populations. For these excess sources, we have to investigate whether they are evolved high-mass stars, or whether the excess emission stems from a circumstellar disk.

Spectroscopically classified evolved, high-mass stars (Martins et al. 2008) are indicated as crosses and asterisks in the two-color diagram. While O4-6 supergiants cluster close to the main sequence population, nitrogen-enriched WN8-9h stars occupy the locus of classical T Tauri stars (CTTSs; Meyer et al. 1997), which represents characteristic colors for young, diskbearing stars. The $L^{\prime}$-band excess of these W-R stars is, however, not caused by disk emission, but originates in extended dusty envelopes produced by their strong stellar winds. As the $L$-band properties of these sources have not been measured previously, we report their photometry in Table 5. Three of the brightest cluster members $(K<11.5 \mathrm{mag})$ not covered by the Martins et al. (2008) spectroscopic survey of evolved stars display colors comparable to the identified WN8-9h stars, indicating that these sources have also started their post-main-sequence evolution. These three sources are aslo included in Table 5. These contaminants in the two-color diagram are found only in massive star clusters, and are not a source of uncertainty in the disk fractions measured in lower-mass star-forming regions. For the purpose of deriving the disk fraction in the Arches cluster (Section 3.2), evolved stars with $K^{\prime}<14$ mag are excluded from the excess sample representing circumstellar disks.

Several very red cluster members with excesses of at least $0.3 \pm 0.02 \mathrm{mag}$ redder in $K^{\prime}-L^{\prime}$ than even the evolved cluster population stand out in the two-color diagram at $K^{\prime}-$ $L^{\prime}>2.3$ mag. While the spectroscopically studied sources in Martins et al. (2008) comprise the brightest part of the cluster main sequence with likely progenitor masses above $60 M_{\odot}$, the comparison with the color-magnitude diagram (CMD; Figure 7) reveals that the extreme excess sources are faint. With $K^{\prime}$-band magnitudes of 14.5-18 mag (Table 6), their stellar 

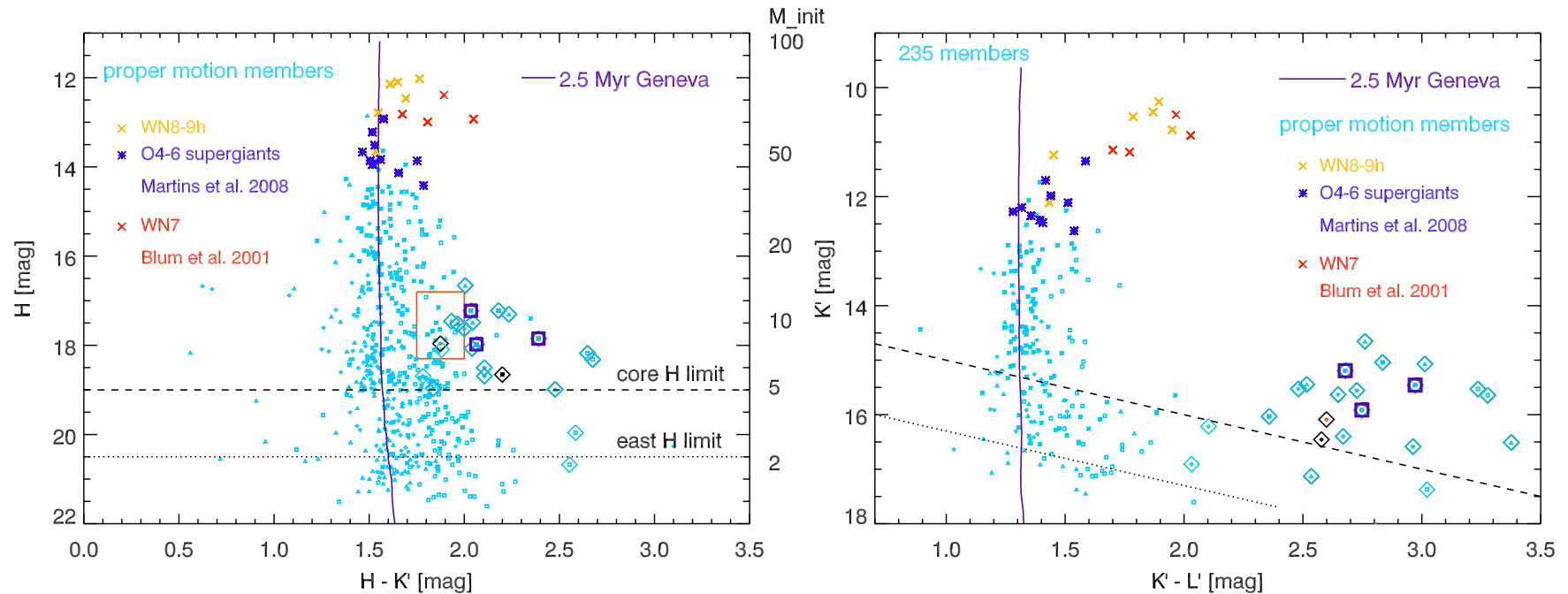

Figure 7. Keck/NIRC2 color-magnitude diagrams (CMDs) of Arches proper motion members. $K^{\prime}-L^{\prime}$ excess sources are labeled (diamond symbols). The dashed lines indicate the completeness limit due to the turnover in the $H$-band LF (left panel) and the $L^{\prime}$-band LF (right panel) in the crowding-limited core and the less dense east fields. A 2.5 Myr Geneva isochrone with solar metallicity, shifted to an adopted distance to the GC of $8 \mathrm{kpc}\left(\mathrm{Ghez}\right.$ et al. 2008) and $A_{V}=26$ mag (Stolte et al. 2005), using the Rieke \& Lebofsky (1985) extinction law measured toward the GC, is shown for reference. The location of red clump stars in the inner bulge is indicated by the red box. The three sources with SINFONI spectroscopy are marked by blue boxes. Left: the $H-K^{\prime}$ vs. $H$ CMD reveals that some $L^{\prime}$-band excess sources blend with the extincted population along the GC line of sight toward the Arches, while several excess sources display significant $K^{\prime}$-band excess as well. Right: In the $K^{\prime}-L^{\prime}$ vs. $K^{\prime}$ CMD, $L^{\prime}$-band excess sources are clearly distinct, displaying offsets of $\geqslant 0.5$ mag from main sequence stars. While contamination with the main sequence and possible red clump interlopers at the GC would be a major problem in $H-K^{\prime}$ without membership information, the ambiguity is resolved in $K^{\prime}-L^{\prime}$.

(A color version of this figure is available in the online journal.)

Table 5

$L$-band Properties of Wolf-Rayet Stars

\begin{tabular}{ccccccccccc}
\hline \hline ID & $\begin{array}{c}\delta \text { R.A. } \\
\left({ }^{\prime \prime}\right)\end{array}$ & $\begin{array}{c}\delta \text { Decl. }^{\mathrm{a}} \\
\left({ }^{\prime \prime}\right)\end{array}$ & $\begin{array}{c}H \\
(\mathrm{mag})\end{array}$ & $\begin{array}{c}\sigma_{H} \\
(\mathrm{mag})\end{array}$ & $\begin{array}{c}K^{\prime} \\
(\mathrm{mag})\end{array}$ & $\begin{array}{c}\sigma_{K} \\
(\mathrm{mag})\end{array}$ & $\begin{array}{c}L^{\prime} \\
(\mathrm{mag})\end{array}$ & $\begin{array}{c}\sigma_{L} \\
(\mathrm{mag})\end{array}$ & $\begin{array}{c}\text { SpT } \\
\text { Ref. }^{\text {b }}\end{array}$ \\
\hline 1 & 0.000 & 0.000 & 12.023 & 0.030 & 10.258 & 0.179 & 8.364 & 0.100 & WN8-9h & 1 \\
2 & 1.936 & 4.725 & 12.097 & 0.044 & 10.447 & 0.090 & 8.577 & 0.101 & WN8-9h & 1 \\
3 & 0.664 & 2.764 & 12.145 & 0.021 & 10.535 & 0.079 & 8.749 & 0.100 & WN8-9h & 1 \\
4 & -0.419 & 1.048 & 12.465 & 0.013 & 10.772 & 0.021 & 8.822 & 0.100 & WN8-9h & 1 \\
5 & 3.381 & -0.302 & 12.819 & 0.014 & 11.144 & 0.011 & 9.443 & 0.100 & WN7/OIf* & 2,3 \\
6 & 1.348 & 1.620 & 12.925 & 0.013 & 11.349 & 0.014 & 9.763 & 0.100 & O4-6If* & 1 \\
7 & -1.893 & 5.057 & 12.992 & 0.025 & 11.185 & 0.025 & 9.414 & 0.100 & WN7 & 2 \\
8 & 4.522 & 8.055 & 12.390 & 0.023 & 10.497 & 0.030 & 8.530 & 0.108 & $\ldots$ & $\ldots$ \\
\hline
\end{tabular}

Notes.

${ }^{a}$ Positional offsets in right ascension and declination are given in arcseconds, relative to the brightest source in the cluster core (see Figure 1), R.A. 17:45:50.42, decl. $-28: 49: 22.3$. The cluster center is located at R.A. 17:45:50.54, decl. $-28: 49: 19.8$.

${ }^{\mathrm{b}}$ References for spectral types: (1) Martins et al. 2008; (2) Blum et al. 2001; (3) Figer et al. 2002.

masses are expected to be substantially lower than W-R stars (Section 3.1.2). As the spectra of three of these sources with large $L^{\prime}$-band excess display strong $\mathrm{CO}$ bandhead emission lines (Section 3.2), their $L^{\prime}$-band excesses most likely originate in circumstellar disks.

Formally, we define disk sources as cluster members with $K^{\prime}-L^{\prime}$ colors too red to be reddened main sequence stars, and that are too faint to be W-R stars $(K>14 \mathrm{mag})$. To distinguish sources with significant $K^{\prime}-L^{\prime}$ excess from the cluster main sequence, we determine the standard deviation in the main sequence population from the rms in the $K^{\prime}-L^{\prime}$ and $H-K^{\prime}$ color of stars with $K^{\prime}-L^{\prime}<1.6 \mathrm{mag}$ to be $\operatorname{rms}_{K L}=0.11 \mathrm{mag}$ and $\mathrm{rms}_{H K}=0.14 \mathrm{mag}$. The $2 \sigma$ ellipse with a major and minor axis of twice the derived standard deviations in $H-K^{\prime}$ and $K^{\prime}-L^{\prime}$, respectively, is shown in the two-color diagram in Figure 6. Note that the apparent circular shape of the ellipse is a consequence of the unequal axis spacing. The tangent lines parallel to the reddening vector (dashed lines) indicate the color regime where cluster stars with higher foreground extinctions can be found. The color variations of sources above the reddening path are dominated by their photometric uncertainties. These randomly scattered sources above the reddening path are enveloped by the dashed lines, indicating that all main sequence members without excess both above and below the reddening path are confined between these $2 \sigma$ limits. Hence, we define stars significantly beyond these $2 \sigma$ limits as excess sources. Specifically, sources to the right of the lower dashed line in Figure 6 are required to meet the following criteria:

$$
\begin{aligned}
& \left(K^{\prime}-L^{\prime}\right)-\sigma_{K^{\prime}-L^{\prime}}>\left(H-K^{\prime}\right) / m_{\mathrm{ext}}+0.37 \\
& \left(H-K^{\prime}\right)+\sigma_{H-K^{\prime}}<m_{\mathrm{ext}} *\left(K^{\prime}-L^{\prime}\right)-m_{\mathrm{ext}} * 0.37,
\end{aligned}
$$

where the slope of the Rieke \& Lebofsky (1985) extinction law is given by

$$
\begin{aligned}
m_{\mathrm{ext}} & =\left(A_{H}-A_{K}\right) /\left(A_{K}-A_{L}\right) \\
& =(0.175-0.112) /(0.112-0.058)=1.1667 .
\end{aligned}
$$


Table 6

$L$-band Excess Sources in the Arches Cluster

\begin{tabular}{|c|c|c|c|c|c|c|c|c|c|c|}
\hline ID & $\begin{array}{c}\delta \text { R.A. } \\
\left({ }^{\prime \prime}\right)\end{array}$ & $\begin{array}{c}\delta \text { Decl. }^{\mathrm{a}} \\
\left({ }^{\prime \prime}\right)\end{array}$ & $\begin{array}{c}H \\
(\mathrm{mag})\end{array}$ & $\begin{array}{c}\sigma_{H} \\
(\mathrm{mag})\end{array}$ & $\begin{array}{c}K^{\prime} \\
(\mathrm{mag})\end{array}$ & $\begin{array}{c}\sigma_{K} \\
(\mathrm{mag})\end{array}$ & $\begin{array}{c}L^{\prime} \\
(\mathrm{mag})\end{array}$ & $\begin{array}{c}\sigma_{L} \\
(\mathrm{mag})\end{array}$ & $\begin{array}{c}A_{V}^{\mathrm{b}} \\
(\mathrm{mag})\end{array}$ & Spectra \\
\hline 1 & 5.852 & 2.239 & 17.220 & 0.059 & 15.040 & 0.011 & 12.205 & 0.103 & $25.4 \pm 2.1$ & \\
\hline 2 & 3.328 & 2.113 & 17.227 & 0.014 & 15.192 & 0.009 & 12.514 & 0.100 & $25.8 \pm 0.5$ & $\mathrm{Sp}$ \\
\hline 3 & -2.948 & 3.563 & 17.848 & 0.064 & 15.458 & 0.010 & 12.486 & 0.103 & $27.4 \pm 0.6$ & $\mathrm{Sp}$ \\
\hline 4 & 3.947 & 1.106 & 17.979 & 0.025 & 15.915 & 0.010 & 13.167 & 0.100 & $25.8 \pm 0.7$ & $\mathrm{Sp}$ \\
\hline 5 & -0.963 & 0.526 & 18.071 & 0.019 & 16.030 & 0.011 & 13.672 & 0.101 & $25.1 \pm 0.7$ & \\
\hline 6 & -2.993 & -0.440 & 18.656 & 0.178 & 16.456 & 0.058 & 13.878 & 0.103 & $25.1 \pm 1.3$ & \\
\hline 7 & 7.064 & -0.835 & 17.521 & 0.022 & 15.555 & 0.018 & 12.828 & 0.104 & $25.5 \pm 1.3$ & \\
\hline 8 & 11.637 & -2.263 & 17.962 & 0.022 & 16.087 & 0.020 & 13.488 & 0.103 & $23.3 \pm 0.5$ & \\
\hline 9 & 7.470 & 2.160 & 18.502 & 0.014 & 16.397 & 0.024 & 13.728 & 0.104 & $24.5 \pm 0.7$ & \\
\hline 10 & 7.850 & 1.769 & 18.095 & 0.020 & 16.215 & 0.016 & 14.112 & 0.102 & $25.5 \pm 1.6$ & \\
\hline 11 & 11.772 & -0.970 & 18.691 & 0.023 & 16.585 & 0.017 & 13.622 & 0.113 & $23.4 \pm 0.7$ & \\
\hline 12 & 14.877 & -1.496 & 17.459 & 0.038 & 15.526 & 0.023 & 13.045 & 0.102 & $23.6 \pm 0.6$ & \\
\hline 13 & 15.480 & -4.183 & 17.630 & 0.026 & 15.630 & 0.023 & 12.982 & 0.102 & $23.7 \pm 1.0$ & \\
\hline 14 & 5.329 & 7.119 & 16.659 & 0.032 & 14.654 & 0.024 & 11.893 & 0.107 & $25.7 \pm 1.2$ & \\
\hline 15 & 3.512 & 15.159 & 17.303 & 0.031 & 15.069 & 0.032 & 12.056 & 0.110 & $25.2 \pm 2.6$ & \\
\hline 16 & 8.788 & 7.212 & 17.487 & 0.029 & 15.443 & 0.017 & 12.927 & 0.107 & $24.8 \pm 1.6$ & \\
\hline 17 & 9.011 & 13.247 & 18.987 & 0.020 & 16.509 & 0.026 & 13.133 & 0.108 & $26.2 \pm 1.9$ & \\
\hline 18 & 2.435 & 14.149 & 18.965 & 0.035 & 17.128 & 0.029 & 14.593 & 0.132 & $24.7 \pm 1.5$ & \\
\hline 19 & -12.619 & 1.492 & 18.177 & 0.026 & 15.529 & 0.013 & 12.293 & 0.101 & $24.8 \pm 2.4$ & \\
\hline 20 & -5.101 & 4.691 & 18.318 & 0.013 & 15.643 & 0.020 & 12.366 & 0.103 & $27.9 \pm 0.6$ & \\
\hline 21 & -4.151 & 4.383 & 19.958 & 0.018 & 17.373 & 0.044 & 14.351 & 0.102 & $27.7 \pm 0.8$ & \\
\hline 22 & -11.824 & 2.234 & 20.676 & 0.021 & 18.123 & 0.009 & 15.317 & 0.116 & $24.8 \pm 2.4$ & \\
\hline 23 & 16.215 & 0.249 & 18.690 & 0.025 & 16.907 & 0.025 & 14.875 & 0.111 & $22.7 \pm 0.6$ & \\
\hline 24 & 4.240 & 2.615 & 17.366 & 0.051 & 15.607 & 0.012 & 13.527 & 0.104 & $26.3 \pm 1.0$ & \\
\hline
\end{tabular}

Notes.

a Positional offsets in right ascension and declination are given in arcseconds, relative to the brightest source in the cluster core (see Figure 1), R.A. 17:45:50.42, decl. $-28: 49: 22.3$. The cluster center is located at R.A. 17:45:50.54, decl. $-28: 49: 19.8$.

b The visual extinction is estimated as an average of the extinction of the four nearest main-sequence $\left(1.4\right.$ mag $<H-K^{\prime}<1.9$ mag) cluster members using a Rieke \& Lebofsky (1985) extinction law.

The $y$ intercept is determined by the shift of the reddening vector to the tangent of the $2 \sigma$ ellipse, $\delta_{2 \sigma}=0.37 . \sigma_{H-K^{\prime}}$ and $\sigma_{K^{\prime}-L^{\prime}}$ denote the photometric color uncertainties of each source. These requirements imply that sources are counted as having an excess when their colors are significantly to the right of the $2 \sigma$ reddening band in $K^{\prime}-L^{\prime}$ and significantly downward of the $2 \sigma$ reddening band in $H-K^{\prime}$. Figure 6 (right) shows the selection of significant excess members as diamonds. The bulk of the excess sample is shown in the insert in Figure 6 (right) with photometric uncertainties, illustrating that the uncertainty in the colors of each excess source cannot account for their observed distances from the main sequence locus and the reddening band. With these selection criteria, $24 L^{\prime}$-band excess sources are detected with $K^{\prime}>14 \mathrm{mag}$. In addition, six stars with $K^{\prime}>14$ mag are located close to the CTTS locus, but are consistent with a locus inside the reddening band when their photometric uncertainties are taken into account. These sources can either be main sequence cluster members suffering enhanced foreground extinction along the line of sight or sources with weaker $K^{\prime}-L^{\prime}$ excesses. As the nature of these stars cannot be unambiguously determined at this point, they are not included in the excess sample. The photometric properties of the $L^{\prime}$-band excess sources are summarized in Table 6 . Of the 24 sources with infrared excess, 23 have measured proper motions (red diamonds in Figure 5), while one star is not resolved in the NACO reference image due to its proximity to a bright neighbor. One source, located at the edge of the NIRC2 FOV, is formally a non-member, but its positional uncertainty in the $x$-direction is a factor of 5 higher than the mean uncertainty in the excess sample and does not allow for a final conclusion on its membership. The second formal non- member is blended with a source of comparable brightness, and the two sources are not well resolved in the NACO $K$-band reference image. All 21 infrared excess sources with reliable proper motion measurements are proper motion members of the Arches cluster. It is extremely unlikely to find 21 sources with specific colors to be cluster members simultaneously. The region in the proper motion plane covered by the majority of the field

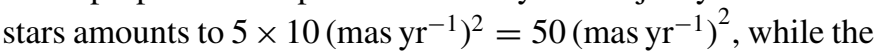
excess sources cover an area of $2 \times 2\left(\operatorname{mas~yr}^{-1}\right)^{2}$, and the full $2 \sigma$ circle is exactly $\pi \cdot 1.28^{2}=5.15\left(\text { mas yr }^{-1}\right)^{2}$. Hence, the likelihood to find a single field source inside the $2 \sigma$ candidate circle is $5.15 / 50=0.10$, and the likelihood to find 21 sources in this area of the proper motion plane simultaneously is only $(0.1)^{21}=10^{-21}$. Given the uncertain proper motion of the three remaining sources with $L^{\prime}$-band excess colors, we consider all 24 sources members of the cluster.

Within the completeness limit in the cluster core, $H<$ $19 \mathrm{mag}$, the $L^{\prime}$-band excess cluster members occupy a magnitude range of $16.6 \mathrm{mag}<H<19.0 \mathrm{mag}$, and span a color range of $2.03 \mathrm{mag}<K^{\prime}-L^{\prime}<3.38 \mathrm{mag}$ (Table 6). The cluster main sequence population covering the same $H$-band magnitudes displays a mean color of $K^{\prime}-L^{\prime}=1.42 \pm 0.11 \mathrm{mag}$ (rms). With a mean color of $K^{\prime}-L^{\prime}=2.73 \pm 0.36 \mathrm{mag}(\mathrm{rms})$, the $19 L^{\prime}$-band excess members with $H<19$ mag are separated from the cluster main sequence by $\Delta\left(K^{\prime}-L^{\prime}\right)=1.31_{-0.70}^{+0.65} \mathrm{mag}$, and hence are redder than the mean main sequence color by at least $0.6 \mathrm{mag}$ (see Figure 7, right panel). The comparison with the $H-K^{\prime}, K^{\prime}$ CMD shows that the excess sources are not revealed by $H K$ photometry alone. In particular, the bluest sources with $L^{\prime}$-band excess emission overlap in the $H-K^{\prime}$, 

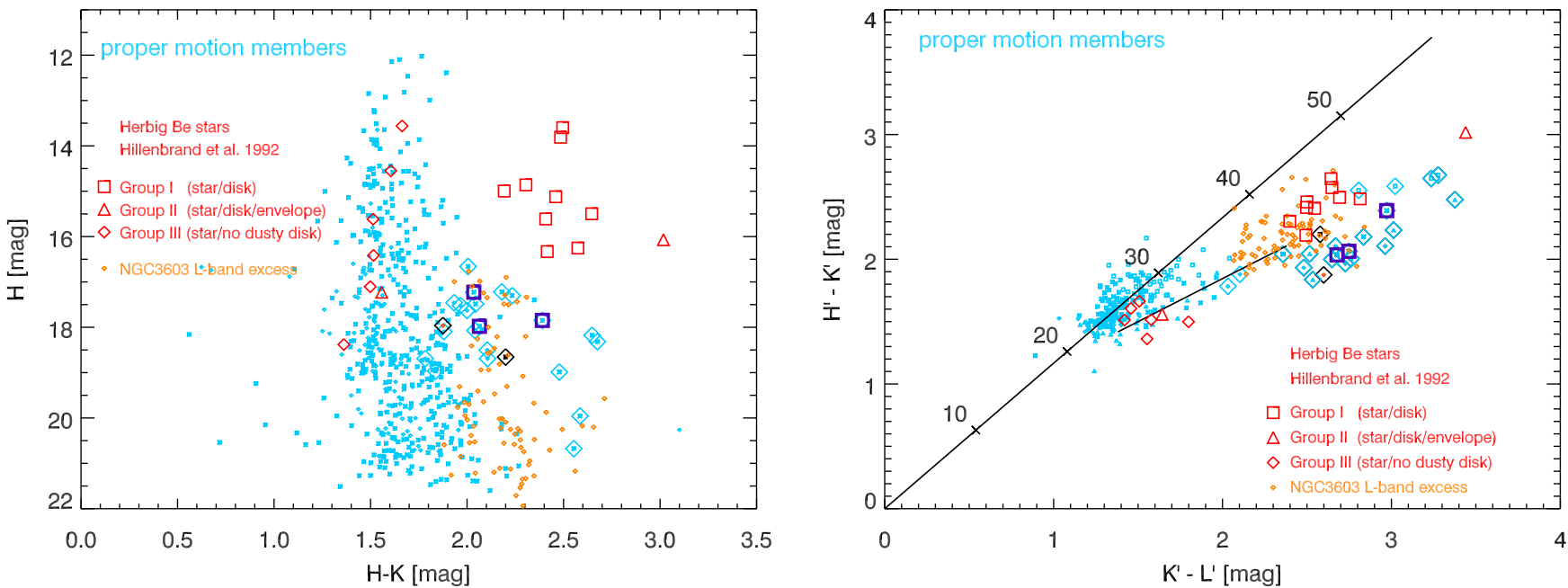

Figure 8. Same as Figures 9 and 8 with Herbig Be disks from Hillenbrand et al. (1992) and NGC $3603 L^{\prime}$ excess sources from Stolte et al. (2004) included. The Herbig Be and NGC 3603 sources were adjusted to the distance and foreground extinction of the Arches cluster. Herbig Be Group I star/disk systems with ages <1 Myr occupy the same $K^{\prime}-L^{\prime}$ color regime as the Arches excess sources, but are systematically brighter. The Arches disks at an age of $2.5 \mathrm{Myr}$ may be similar star/disk systems at a later evolutionary state.

(A color version of this figure is available in the online journal.)

$K^{\prime}$ CMD with the color-magnitude location of the red clump in the nuclear bulge $\left(K^{\prime}>15.3 \mathrm{mag}, H-K^{\prime}>1.8 \mathrm{mag}\right.$, for $A_{K} \geqslant 2.4 \mathrm{mag}, M_{K}=-1.6 \mathrm{mag} ;$ Alves 2000). The empirical location of red clump stars stands out in our non-member sample as a clustering at $H=17.5, H-K=1.87 \mathrm{mag}$ and is marked with a red box in the CMDs. The ambiguity between excess sources and red clump stars is resolved in $K^{\prime}-L^{\prime}$, emphasizing the importance of $L$-band observations to derive disk fractions in young star clusters. The completeness limits indicate that the detection of fainter excess candidates is limited by the current $L^{\prime}$-band completeness limit of $L^{\prime}=13.5-14.5 \mathrm{mag}$ in each field.

In summary, we detect 24 sources with significant $L^{\prime}$-band excess, of which all 21 excess sources with reliable membership information are proper motion members of the Arches.

\subsubsection{Spectral Types and Stellar Masses of Disk Host Stars}

In the Arches cluster, photospheric flux measurements of excess sources at visible wavelengths are prohibited by the large foreground extinction toward the GC. Hillenbrand et al. (1992) provide evidence that photospheric emission dominates the spectral energy distribution (SED) of Herbig Be stars below $1.2 \mu \mathrm{m}$, i.e., $J$ band. Even in $J$-band observations, however, most fainter sources are veiled by the foreground extinction of 22-30 mag toward the Arches (Stolte et al. 2002). The $H-K^{\prime}, H$ CMD (Figure 8) shows that the near-infrared excess in $H-K^{\prime}$ is substantially smaller than in $K^{\prime}-L^{\prime}$. The Arches excess sources display on average about $0.5 \mathrm{mag}$ less $H-K^{\prime}$ excess than the Hillenbrand Herbig Be sample, shown as box points. A lower excess at smaller wavelength indicates that the hot, inner disk rim becomes increasingly depleted at these ages, possibly due to the existence of a growing inner hole. As a consequence, the $H$-band flux of the excess sources will be closer to the expected main sequence flux than in younger excess populations. Hence, we use the $H$-band magnitude to derive an approximate mass range for the excess population.

The $H$-band magnitudes are compared to a solar-metallicity, $2.5 \mathrm{Myr}$ main sequence isochrone from the standard set of Geneva stellar evolution models (Lejeune \& Schaerer 2001). The foreground extinction to each excess source (Table 6) is measured from the $H-K^{\prime}, K^{\prime}-L^{\prime}$ color of the four nearest cluster members without excess emission using a Rieke \& Lebofsky (1985) extinction law. The variation in visual extinction ranges from 22.7 to $27.9 \mathrm{mag}$, or $A_{K}=2.5-3.1 \mathrm{mag}$. The variation between individual neighboring sources used to estimate the average extinction on the line of sight to each excess source is large, yielding standard deviations of up to $\sigma_{\mathrm{AV}}=2.4 \mathrm{mag}$. The bright stars concentrated toward the cluster center have blown a cavity evidenced by a lower reddening in the cluster core (Stolte et al. 2002). The mean foreground extinction toward the cluster center is therefore determined from the upper main sequence, $12 \mathrm{mag}<K^{\prime}<15 \mathrm{mag}$ and $1.2 \mathrm{mag}<K^{\prime}-L^{\prime}<1.5 \mathrm{mag}$, which is least biased by variable extinction (see Figure 7), to $A_{V \mathrm{~ms}}=25.2 \pm 1.8$. This value is identical to the mean foreground extinction of $A_{V}=25.3 \pm 1.4 \mathrm{mag}$ of the excess sample. As substantial small-scale spatial variation leads to the large uncertainties in the extinction of each excess source, we use the average extinction toward the excess sample for stellar mass computation. The extinction of $A_{V}=25.3 \mathrm{mag}$ and a distance modulus of $\mathrm{DM}=14.52 \mathrm{mag}(8 \mathrm{kpc})$ are applied to the isochrone $H$ magnitude. No color transformation to the Mauna Kea system was applied, as the difference is smaller than 0.05 mag (Carpenter 2001; Hawarden et al. 2001). The 19 excess sources above the core $H$-band completeness limit are observed within a magnitude range of $16.7 \mathrm{mag}<H<19 \mathrm{mag}$, corresponding to a mass interval of $15 M_{\odot}>M>5 M_{\odot}$. On the zero-age main sequence (ZAMS), these masses correspond to spectral types B1V-B6V. Two $L^{\prime}$ excess sources with $H=19.8$ and $H=20.7$ are not included in the complete disk sample, and the comparison of their faint $H$-band magnitudes with a Geneva $2.5 \mathrm{Myr}$ isochrone indicates that disk-bearing A stars (Herbig Ae stars) with masses down to at least $2.2 M_{\odot}$ exist in the Arches cluster. Likewise, when the maximum foreground extinction uncertainty of $\sigma_{\mathrm{AV}}=2.4 \mathrm{mag}$, corresponding to $\sigma_{\mathrm{AH}}=0.42 \mathrm{mag}$, is taken into account, the full allowable mass range could be as large as $1.8<M / M_{\odot}<18$. A similar lower-mass limit is obtained by allowing for an unaccounted residual $H$-band excess, which might influence the luminosities of the reddest excess sources in the sample, which have $H-K^{\prime}$ colors similar to the Hillenbrand 

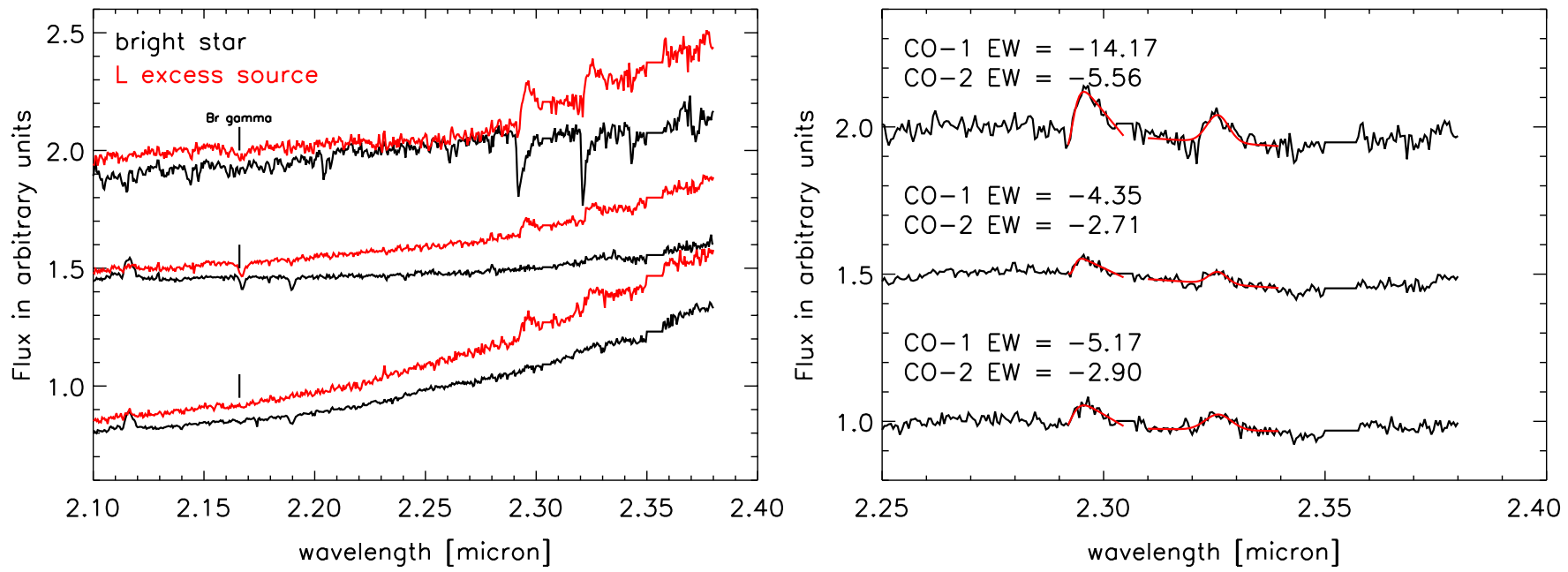

Figure 9. VLT/SINFONI $K$-band spectra of three of the Arches $L^{\prime}$ excess sources. The black lines are spectra of the nearest bright stellar neighbor, while the red lines are spectra of the excess sources. While the spectra of neighboring bright sources show stellar emission and absorption lines, the excess source spectra are dominated by CO bandhead emission at $2.29 \mu \mathrm{m}$ and $2.32 \mu \mathrm{m}$. The right panel shows a continuum-subtracted excerpt of the CO bandhead emission region. The comparison between the steep decrease in $\mathrm{CO}$ absorption of the coincidental background giant neighbor (topmost black spectrum) and the slow rise in the blue wing of the $\mathrm{CO}$ emission in the excess sources provides evidence for rotational broadening. This broadening indicates that the $\mathrm{CO}$ emission arises in the inner parts of rotating circumstellar disks (Bik \& Thi 2004). High-resolution spectroscopy will be required to quantify the rotational velocities in the circumstellar disks. Two of the excess sources display $\mathrm{Br} \gamma$ in absorption, indicating that the extended, massive disks with strong $\mathrm{Br} \gamma$ emission as observed toward younger star-forming regions are already depleted in these objects.

(A color version of this figure is available in the online journal.)

Group I star/disk systems. Characteristic $H$-band excesses in very young Herbig Be stars are in the range $0.3-1$ mag (see, e.g., Figure 2 in the Herbig Ae/Be survey of Hernández et al. 2005). If we allow for a maximum $H$-band excess of 1 mag in the two reddest excess sources, these sources could also have masses as low as $3 M_{\odot}$. At the same time, a small fraction of the disks should be seen edge-on, and for these sources local extinction in the optically thick disk causes additional dimming of the $H$-band flux. A circumstellar visual extinction of $10 \mathrm{mag}$ corresponds to 1.7 mag in $H$ (Rieke \& Lebofsky 1985), such that some of the excess sources could be intrinsically brighter with masses in excess of $20 M_{\odot}$.

In summary, the $H$-band luminosities of the excess population indicate that several $\mathrm{A} 5 \mathrm{~V}$ to B0V stars within a maximum mass range of $2 M_{\odot}<M<20 M_{\odot}$ feature disks in the Arches cluster.

\subsection{Spectroscopic Evidence for Circumstellar Disks}

\subsubsection{CO Bandhead Emission and Evidence for Disk Rotation}

$K^{\prime}$-band spectra of three of the $24 L^{\prime}$-band excess sources are shown in Figure 9. The red lines are the extracted spectra of the excess sources from the SINFONI data cubes, while the black spectra are bright stars located in the immediate vicinity of each excess source. All three excess sources display strong $\mathrm{CO}$ bandhead emission with the first and second overtones at $2.29 \mu \mathrm{m}$ and $2.32 \mu \mathrm{m}$, respectively. The first two overtones are detected at high S/N's of 34-118, where the first overtone is always stronger by approximately a factor of 2 as compared to the second overtone emission.

$\mathrm{CO}$ bandhead emission in high-mass young stellar objects (YSOs) with spectral types O6-B5 are observed in NGC 3576 at ages below 1-2 Myr (Figuerêdo et al. 2002; Blum et al. 2004 ) and in the very young < 1 Myr cluster NGC 6618 in M17 (Hanson et al. 1997; Hoffmeister et al. 2006). There is increasing evidence that the $\mathrm{CO}$ bandhead emission originates in rotating disks. In agreement with previous disk models (Chandler et al. 1995; Bik \& Thi 2004; Blum et al. 2004); Bik et al. (2006) conclude that the $\mathrm{CO}$ bandhead emission is produced in the warm (1500-4500 K), dense, and optically thick region within a few $\mathrm{AU}$ from the central star. The column densities required to model the $\mathrm{CO}$ line profiles in high-resolution spectra, $N(\mathrm{CO}) \geqslant$ $10^{20} \mathrm{~cm}^{-2}$ (Kraus et al. 2000; Bik \& Thi 2004), suggest that $\mathrm{CO}$ molecules survive at these radii as a consequence of selfshielding (Bik \& Thi 2004, and references therein). Blum et al. (2004) and Bik \& Thi (2004) showed that excellent fits to the ro-vibrational CO 2-0 bandhead emission $\left(\lambda_{0}=2.2935 \mu \mathrm{m}\right)$ are obtained with rotating disk models, which particularly well account for the slope of the blue wing of the CO overtones, as well as the redshifted peak. Wind models from stellar or disk winds around young, massive objects, on the other hand, predict a vertical blue edge to the overtone emission band and thus cannot account for the observed slow rise in the blue wing (see Kraus et al. 2000 for a detailed comparison of these scenarios). High-spectral resolution observations of the blue emission wing in the first $\mathrm{CO}$ overtone lent increasing support to the rotating disk interpretation. In particular, Blum et al. (2004) model the CO bandhead emission of four high-mass $\left(5-17 M_{\odot}\right)$ YSOs as Keplerian disks originating in the inner $1 \mathrm{AU}$ from the central star with rotation velocities between 25 and $260 \mathrm{~km} \mathrm{~s}^{-1}$. Spectral models by Bik \& Thi (2004) reveal that the CO bandhead emission originates in the inner parts of a thick accretion disk at radii $0.1-5 \mathrm{AU}$.

The source with the strongest CO bandhead emission (top spectrum in the right panel of Figure 9) displays a slanted ascent in the blue wing of the first overtone. The slope in the blue wing is particularly evident in comparison with the steep drop of the $\mathrm{CO}$ absorption in the nearby background giant, where the envelope emission shows no signs of rotation. The shape of the blue wing is very similar to the Keplerian disks modeled in Bik \& Thi (2004). Although the moderate spectral resolution prohibits the derivation of the rotational velocities of the Arches excess sources, all six detected band heads are consistent with a 


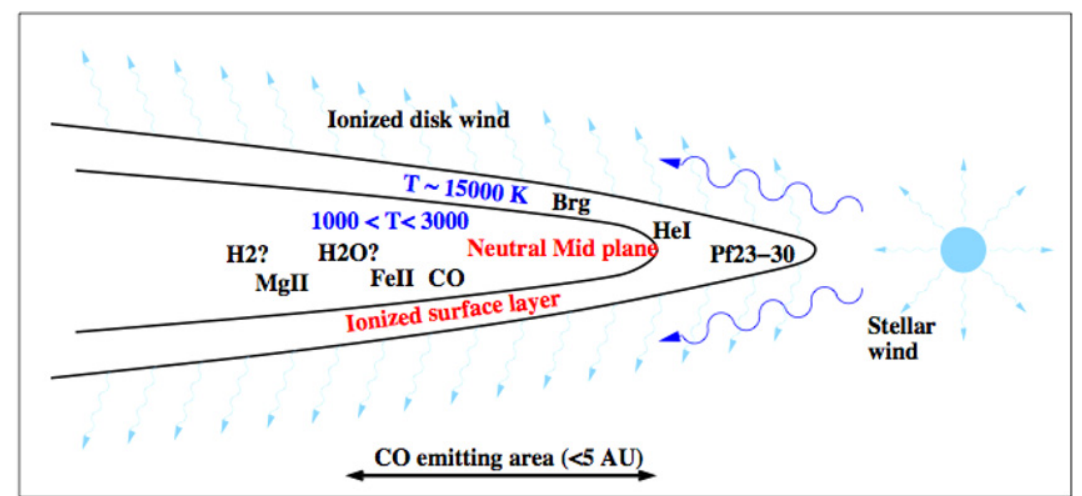

Figure 10. Schematic disk model indicating the zones around a massive young stellar object where CO and ionized gas emission might originate (adopted from Bik et al. 2005). The $\mathrm{CO}$ bandhead emission originates in the dense, self-shielded region within $5 \mathrm{AU}$ from the central star, while Br $\gamma$ emission can be generated in the inner disk rim very close to the star as well as in the illuminated disk surface at all radii if the star emits sufficient $\mathrm{UV}$ radiation to ionize hydrogen. The lack of Br $\gamma$ emission indicates that the disk-bearing stars have spectral types later than B3V.

(A color version of this figure is available in the online journal.)

slow increase in the blue wing. We therefore conclude that the $\mathrm{CO}$ bandhead emission originates in the optically thick inner parts of rotating circumstellar disks.

In addition to $\mathrm{CO}$ emission, two of the three excess source spectra display a weak $\mathrm{Br} \gamma$ absorption feature. Circumstellar disks in young $\mathrm{OB}$ associations typically show strong $\mathrm{Br} \gamma$ emission (e.g., Hanson et al. 1997; Bik et al. 2006). ${ }^{8}$ In particular, the gas around high-mass stars earlier than $\mathrm{B} 3 \mathrm{~V}$ is expected to be ionized by the EUV radiation from the central star. As indicated in the schematic model of a circumstellar disk around a massive protostar (Figure 10, adopted from Bik et al. 2005), Br $\gamma$ emission is expected from the inner disk rim as well as from the illuminated disk surface. The spectral types of the disk-bearing stars with spectra are therefore likely later than B3V.

The increasing evidence of rotating disks around high-mass stars indicates that late $\mathrm{O}$ and early $\mathrm{B}$ stars form via disk accretion in a similar fashion to their low-mass counterparts (where $\mathrm{CO}$ bandhead emission is also interpreted as evidence for thick accretion disks, e.g., Carr et al. 1993; Chandler et al. 1995), which has a profound impact on the paradigm of highmass star formation.

\subsection{Disk Fraction and Radial Dependence}

\subsubsection{Disk Fraction}

With the conclusion that the excess sources in the Arches cluster are dense circumstellar disks, and the membership information from proper motions, we can estimate a robust disk fraction among the B-star population of the Arches cluster. Given that all $19 L^{\prime}$-band excess sources in the complete, $H<19$ mag, sample with precise proper motion information are cluster members, we have concluded in Section 3.1.1 on the basis of their exceptional colors that all 22 sources with $L^{\prime}$-band excess and $H<19$ mag belong to the young Arches population. The main sequence population, on the other hand, is contaminated by foreground and background stars. Hence, we only consider proper motion members in both the excess and main sequence samples to determine the cluster disk fraction.

The $H$-band completeness limit in the cluster core is the most stringent limitation for the detection of main sequence stars. The

\footnotetext{
8 Note that the more evolved classical Be stars and B[e] supergiants also show near-infrared excess, yet are characterized by strong hydrogen emission lines. These objects are superluminous as compared to their main sequence B-type counterparts, while the excess sources in the Arches cluster are comparably faint.
}

corresponding population of main sequence cluster members with $H<19$ mag detected in all three filters is additionally limited by the $L^{\prime}$-band detection limit. Completeness limits of 13.5-14.5 mag imply that only early B-type stars with masses in excess of $\sim 12 M_{\odot}$ can be detected on the main sequence, where excess emission is absent. The stellar mass range indicated for the $L^{\prime}$-band excess sources is therefore largely below the $L^{\prime}$ detection limit of diskless main sequence cluster stars, such that the reference main sequence sample is incomplete. The fraction of sources with $L^{\prime}$-band excess (hereafter excess fraction), defined as the ratio of sources with $L^{\prime}$-band excess over all cluster members with and without excess, $f_{\text {exc }}=N_{L \text {-exc }} /\left(N_{\text {mainseq }}+N_{L \text {-exc }}\right)$, would yield an upper limit to the disk fraction. This limitation can be mitigated by not requiring main sequence stars to be detected in $L^{\prime}$, which increases the main sequence sample from $216 H K^{\prime} L^{\prime}$ detections to a total of 311 cluster members with $H<19 \mathrm{mag}$ and $H-K^{\prime}<2.0 \mathrm{mag}$. The six excess sources falling into the main sequence $H-K^{\prime}$ color regime are excluded from the $H-K^{\prime}$ main sequence sample. Five stars are substantially bluer than the Arches main sequence and may be foreground interlopers which incidentally have similar proper motions. These stars with $H-K^{\prime}<1.2 \mathrm{mag}$ were also rejected. The remaining spread in color in the main sequence sample is caused by extinction variations. This selection leaves us with a main sequence reference sample of 300 sources.

Excluding the three excess sources without (reliable) membership information, and the two sources beyond the $H$-band completeness limit in the core, $H=19 \mathrm{mag}$, the comparison sample of excess sources as defined in Section 3.1.1, contains 19 stars, indicating a disk fraction of $19 /(300+19)=6.0 \% \pm 1.6 \%$ among B-type stars in the Arches cluster.

\subsubsection{Radial Distribution of Excess Sources}

As detailed in Section 1, the dense environment of a starburst cluster is likely to destroy disks more rapidly than low-density star-forming regions sparse in OB stars. This is particularly the case in the compact core of the cluster, where the exceptionally high density of more than $10^{5} M_{\odot} \mathrm{pc}^{-3}$ could lead to significant destruction of disks in the immediate cluster center as compared to larger distances from the core.

With a total of $19 L^{\prime}$-band excess sources in the complete sample, we can begin to investigate the radial distribution of candidate disks in the Arches cluster. In Figure 11, the 

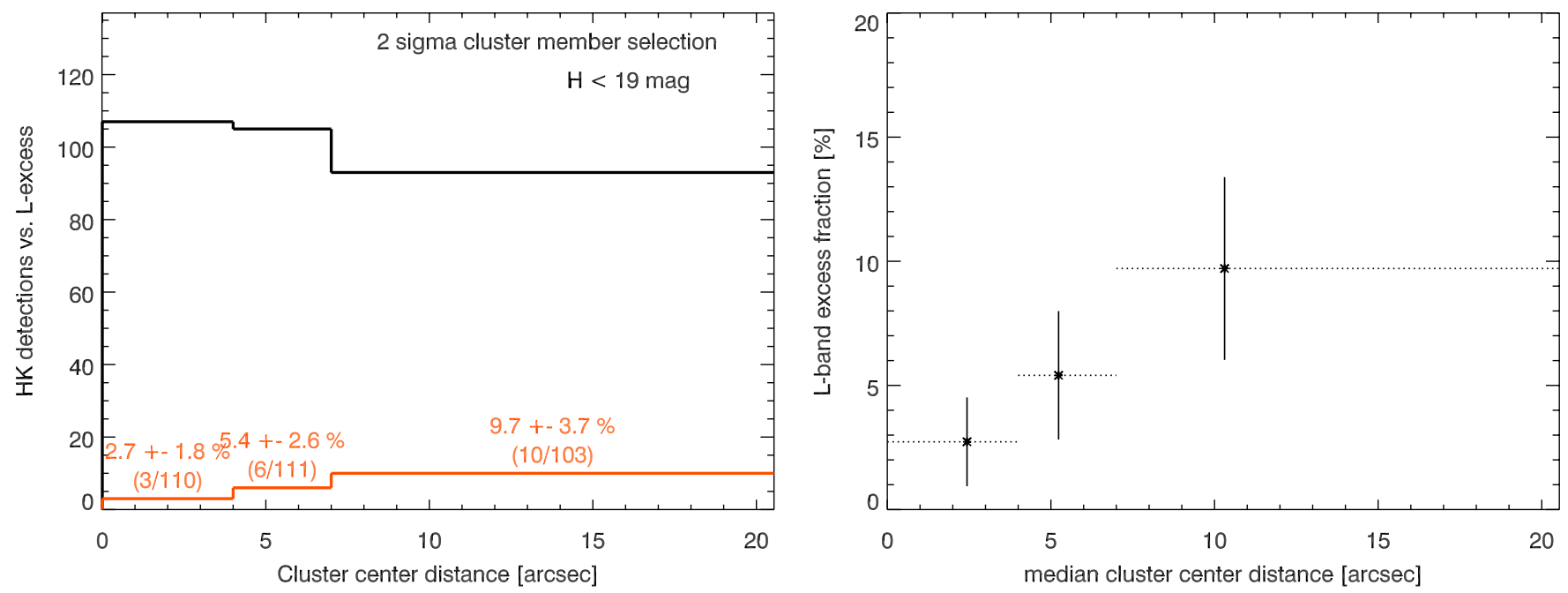

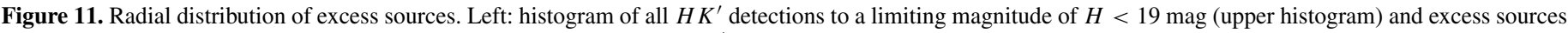

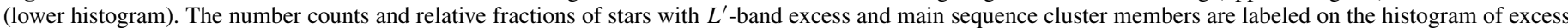

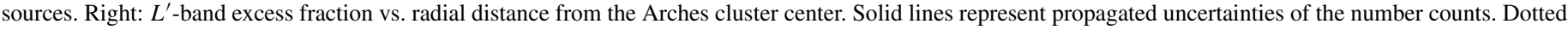

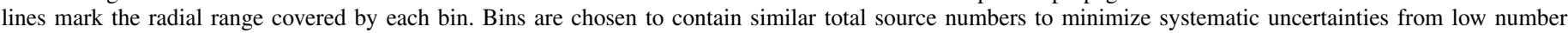
statistics.

(A color version of this figure is available in the online journal.)

histograms of main sequence cluster members with $H<19$ mag without excess (top solid line) and of members with $L^{\prime}$-band excess (bottom solid line) are shown. Radial bins are chosen to contain similar total number counts to minimize systematic uncertainties. The relative fraction of excess sources with respect to main sequence members is labeled, along with the propagated uncertainty. In addition, the absolute number counts of main sequence members with and without excess are given. The right panel of Figure 11 displays the excess fractions versus radial distance from the Arches cluster center. The substantial uncertainties shown as solid lines originate from the low number of excess sources in each bin. The cluster center distance corresponds to the median of main sequence stars in each bin; the radial range covered is indicated by dotted lines. Despite the large statistical uncertainties, there is a strong indication that the disk fraction is significantly lower in the cluster core than at larger radii.

Before concluding that the excess fraction is depleted in the cluster core, incompleteness effects due to the higher stellar density have to be excluded as the source of the observed trend in the disk fraction. Artificial star simulations were carried out in the magnitude range $16 \mathrm{mag}<H<21 \mathrm{mag}$ to probe the completeness of the main sequence sample, and $12 \mathrm{mag}<L<16$ mag to test the disk sample for incompleteness effects. A maximum of 50 artificial stars were randomly inserted in the images to preserve the original stellar density. One-hundred such frames were created in each field, leading to a total of approximately 5000 artificial stars in the probed magnitude ranges. The results of these tests are shown in Figure 12. The histograms (left) show the inserted (thin lines) and recovered source counts (thick lines) in $L^{\prime}$ and $H$. The asterisks in the $L^{\prime}$ histograms show the faintest disk source in each of the three fields displayed. The right panel displays the recovery fractions versus magnitude for $L^{\prime}$ and $H$. The completeness fractions are above $85 \%$ in the outer fields east 1 and halo, and drop to $80 \%$ in the cluster core in the faintest included magnitude bin, for $14 \mathrm{mag}<L^{\prime}<15 \mathrm{mag}$ and $18 \mathrm{mag}<H<19 \mathrm{mag}$. The completeness fractions are comparable on the main sequence and in the disk sample. Only very few sources are actually located in

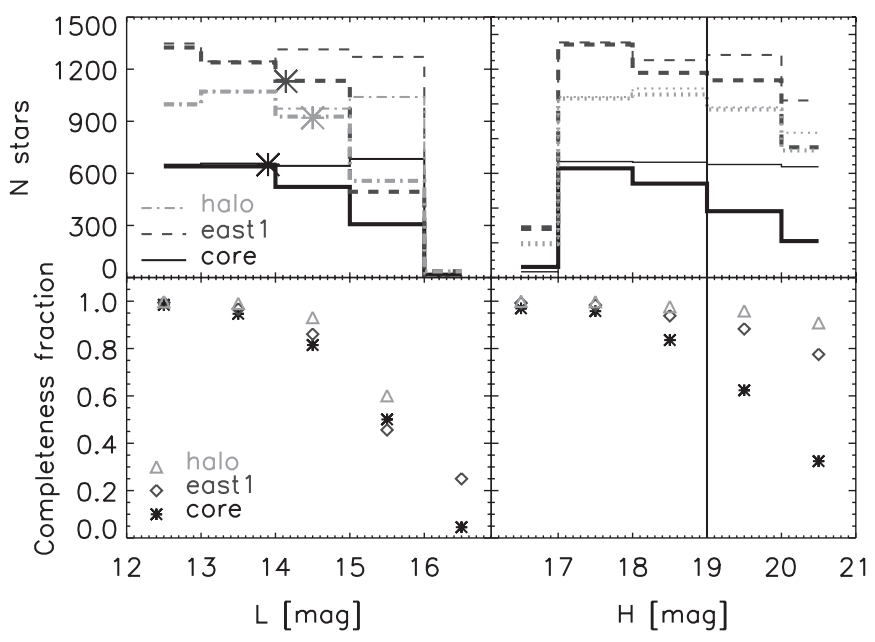

Figure 12. Completeness simulation results in fields core, east1, and halo. The observing conditions are similar for east 1 and east2, while the halo field is representative of the leading field. Top: $L^{\prime}$ and $H$ recovery number counts. Thin lines represent the number counts of inserted artificial stars in each magnitude bin, while thick lines represent the number counts of recovered sources. Asterisks in the $L^{\prime}$ plot represent the faintest excess source in each field. Bottom: $L^{\prime}$ and $H$ completeness fractions. The line at $H=19$ mag shows the imposed $H$-band completeness limit of both main sequence stars and excess sources.

these faint magnitude bins; in the most crowding-limited cluster core sample, only 10 out of 208 main sequence sources are found in the faint bin, such that the $20 \%$ correction adds a mere two sources to the core main sequence sample. Similarly, all disks in the cluster core are brighter than $L^{\prime}=14 \mathrm{mag}$, although the simulation readily detects sources down to $L^{\prime}=15 \mathrm{mag}$. As crowding is not affecting detections in the outer fields, the incompleteness effects are entirely negligible at larger radii, as expected. As a consequence, even when we account for incompleteness, the disk fractions at all radii do not change appreciably.

In conclusion, the disk fraction in the Arches cluster increases significantly from $2.7 \%$ in the cluster core, with $r<0.16 \mathrm{pc}$, to $9.7 \%$ at $r>0.3 \mathrm{pc}$. 


\section{DISCUSSION}

\subsection{Disks in the Arches Cluster}

The finding of disks in the Arches B-star population is unexpected for two reasons. First, disk depletion depends on the UV radiation of the host star. The $H$-band brightness of the disk-bearing stars suggests that the majority are B-type stars. As the characteristic UV evaporation timescale of a primordial disk around Herbig Be stars is less than $1 \mathrm{Myr}$ (Alonso-Albi et al. 2009), a disk lifetime of $2.5 \mathrm{Myr}$ for B-type stars implies that the disks had to be massive initially. Second, disk destruction is expected to be accelerated in a starburst cluster environment. The extreme UV radiation from numerous O-stars adds to the evaporation by the disk host star. At a density of $10^{5} M_{\odot} \mathrm{pc}^{-3}$, interactions cause additional tidal truncation especially of the extended outer disks (Olczak et al. 2008), which in turn enhances the exposure of the inner disk to the cluster radiation field. The detection of optically thick disks with substantial $L$-band excesses close to the cluster center suggests that the disk-bearing sources have migrated into the sphere of influence of the central O-type stars only recently, or are viewed in projection toward the cluster center. The radial increase of the disk fraction toward larger cluster center distances evidences the influence of the radiation field and high stellar density in the cluster core on primordial disks. Speculatively, one can envision a scenario wherein disk-bearing stars migrate inward during the mass segregation process and once close to the cluster center lose their disks rapidly. Taking into account that some of the core sources might be in front of or behind the cluster center, the very low excess fraction of only $3 \%$ in the cluster core is already an upper limit to the disk fraction inside the core radius of $0.2 \mathrm{pc}$. The disk fraction of $10 \%$ for stars with $r>7^{\prime \prime}$ $(0.28 \mathrm{pc})$ in the same stellar mass range provides stringent evidence that the starburst cluster environment enhances the depletion of primordial disks as soon as their orbital motion moves disk-bearing stars close to the cluster center. In the future, the increasing proper motion accuracy might provide trajectories of these sources to understand their orbital motion in the cluster.

From observations of lower-mass star-forming regions, we might expect an increase in the disk fraction toward stars with later types, such as AFG stars. If the same processes dominated disk destruction in a starburst cluster, a substantial increase in the disk fraction in fields with deeper $L$-band observations should be observed, which is not the case. For instance, in the leading field with a peak in the LF of $L^{\prime}=15.5 \mathrm{mag}$, the same number of five excess sources is observed as in the shallower east1 and core fields with $L^{\prime}$ peak luminosities of only 13.5 and 14.0 mag. If the disk fraction would mostly be determined by the UV evaporation from the B-type host stars, then the deeper detection limit in the leading field should yield a significantly larger fraction of disks than the shallower fields, especially when compared to the east1 field with similar stellar density. The fact that an increase in the disk fraction toward fainter stars seems not to be observed in the Arches cluster strengthens the interpretation that the starburst environment affects disk survival. It appears that stars later than B lose their disks more rapidly in a starburst cluster environment than in moderate starforming regions, indicating that the influence of the ambient UV radiation field and encounters indeed shorten the disk survival timescale.

In Figure 8, the Arches population is compared to disks from the most extensive Herbig $\mathrm{Ae} / \mathrm{Be}$ survey of Hillenbrand et al. (1992). The Herbig Be star disks were shifted to the distance and foreground extinction of the Arches cluster. The Arches sources are systematically fainter than the primordial Group I star/ disk systems in the Hillenbrand sample, which is the youngest group with ages of a few $10^{5}$ Myr. In addition, the Arches disks cover $H-K^{\prime}$ colors closer to the main sequence than primordial Herbig Be disks, while still displaying significantly larger excesses than the Group III sources with depleted disks in the Hillenbrand sample. Also included in the CMD are $L$-band excess sources in NGC 3603 at an age of 1 Myr (Stolte et al. 2004; Harayama et al. 2008). The brightest disks in NGC 3603 are comparable in color and magnitude to the Arches sources. The difference between the disks in the Arches and NGC 3603, compared to the primordial disks studied by Hillenbrand et al. (1992), illustrates that the transition from primordial, optically thick dusty disks to evolved disks subject to evaporation and grain growth is a rapid process that happens at an age of approximately $1-2 \mathrm{Myr}$.

\subsection{Comparison with Other Young, Massive Clusters}

One of the best studied, nearby star-forming regions sufficiently massive to host O-type stars is the ONC with a disk fraction of $78 \%$ in the predominantly low-mass population (Lada et al. 2000). The finding of significantly different disk fractions for high- and low-mass stars (80\% for low-mass stars with $M<2 M_{\odot}, 42 \%$ for OBA stars) in this study corroborates the dependence of the disk depletion timescale on the mass of the central star. The ONC provides a very early stage of a massive cluster at an age of $1 \mathrm{Myr}$, and allows predictions of the effects of close encounters with the UV radiating O-star. Dynamical simulations of disk evaporation in the ONC suggest that, while outer disks are depleted, the inner disk is capable of surviving out to $10 \mathrm{AU}$ in the immediate vicinity of the central O6 star $\theta^{1} \mathrm{C}$ Ori (Scally \& Clarke 2001). The possibility of survival of dense, inner disks around B-type stars up to ages of $10 \mathrm{Myr}$ was also predicted theoretically from hydrodynamic simulations taking into account various levels of photoevaporation, disk-stellar wind interaction, mass loss from disk winds, and the evaporative depletion by nearby O-stars (Richling \& Yorke 2000; see Hollenbach et al. 2000; Zinnecker \& Yorke 2007 for reviews).

At an age of $2.5 \mathrm{Myr}$, the Arches cluster fills a rarely sampled gap between the very young UCHII regions and deeply embedded clusters and the more evolved open clusters. As one of the most massive young, compact star clusters in the Milky Way today, it is one of the rare loci where stars with masses above $100 M_{\odot}$ were formed. With a population of at least 125 O-type stars (Section 1), the cluster UV radiation field of $\sim 4 \times 10^{51}$ photons s ${ }^{-1}$ is intense (Lang et al. 2001). When comparing the disk fraction in the Arches with moderate star-forming regions, where disk fractions are studied among lower to intermediatemass stars $\left(\sim 0.5-10 M_{\odot}\right)$, the major problem is to distinguish the effects of the mass dependence and the environment.

A comprehensive survey of Herbig Ae/Be stars in nearby OB associations was carried out by Hernández et al. (2005). In the six star-forming regions with ages 3-16 Myr, these authors find that only a small fraction, $0 \%-5 \%$ of intermediate-mass stars $\left(1.4 M_{\odot}<M<15 M_{\odot}\right)$, are in the Herbig Ae/Be phase, which they interpret as the fraction of intermediate-mass stars having retained dusty inner disks, in agreement with our findings above. From the comparison of disk frequencies around low $\left(M<1 M_{\odot}\right)$ to intermediate-mass stars, Hernández et al. (2005) conclude that at an age of $3 \mathrm{Myr}$, the fraction of disks around intermediate-mass stars is 10 times lower than around their lowmass counterparts. 


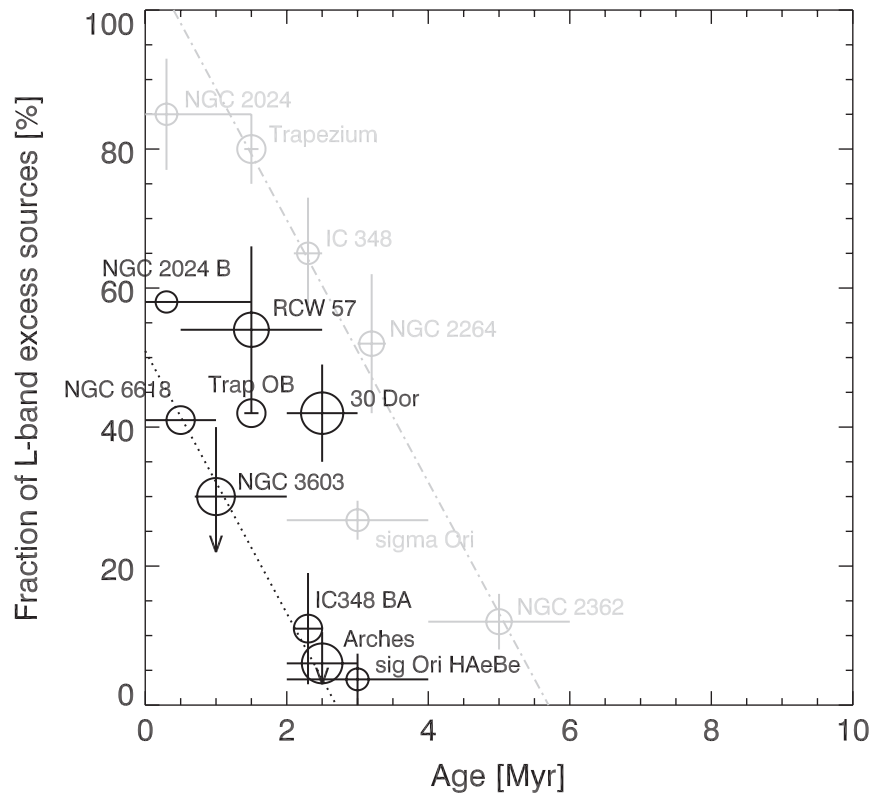

Figure 13. Disk fraction vs. cluster age reproduced from Haisch et al. (2001), including data points from Hernández et al. (2007; sigma Ori), Hoffmeister et al. (2006; M17: NGC 6618), Maercker \& Burton (2005; 30 Dor region), and Maercker et al. (2006; NGC 3576: RCW 57). Symbols are scaled to the logarithm of the cluster mass, from the least massive with $\sim 200 M_{\odot}$ in stars (NGC 2024 IC $348, \sigma$ Ori), to the most massive with $>30,000 M_{\odot}$ (30 Dor; see Table 7). Black circles mark disk fractions derived from high-mass stars of types OBA only, while light gray circles mark populations dominated by low-mass stars. Note that the 30 Dor disk fraction covers the extended H II region, including star-forming ridges harboring YSO candidates, but does not resolve the central cluster, and is therefore an upper limit to the disk fraction in this environment. The dash-dotted line corresponds to the linear decrease in disk fraction vs. cluster age as fitted by Haisch et al. (lighter circles only). In the case of the Arches and NGC 3603, the downward arrow indicates the radial decrease in the fraction of disks from larger radii toward the cluster core (NGC 3603 outer cluster region: Stolte et al. 2004; resolved core: Harayama et al. 2008). The dotted line is a parallel shift from the linear fit by Haisch et al. Intriguingly, there appear to be several populations of clusters following a similarly steep decline in disks, albeit from different initial disk fractions, suggesting a strong environmental effect on disk survival. This sample is comprised of a heterogeneous set of clusters over a large distance and cluster mass scale, probing disks in different stellar mass regimes in addition to the cluster environment. The difference in the disk survival timescales between moderate- and high-mass clusters reveals the necessity to probe the low-mass population in massive clusters to distinguish environmental effects from the stellar mass dependence of the disk lifetime.

In Figure 13, we reproduce the disk fraction versus age diagram for nearby star-forming regions from Haisch et al. (2001), including recent results on a variety of clusters, in addition to the Arches and NGC 3603 data points. The cluster properties and literature references are summarized in Table 7. At cluster ages similar to the Arches, the $\sigma$ Orionis cluster at an age of 3 Myr displays a total thick disk fraction of $27 \% \pm 3 \%$ for all stars $>0.1 M_{\odot}$ (including some brown dwarfs), but this fraction drops to $4 \% \pm 4 \%$ for stars with masses above $2 M_{\odot}$ (Herbig Ae/Be; Hernández et al. 2007). Especially this latter fraction for intermediate-mass stars is strikingly similar to the Arches excess fraction. The central 30 Doradus star-forming region with a mean age range of 2-3 Myr displays an $L$-band excess fraction of $42 \% \pm 5 \%$ (Maercker \& Burton 2005), with indications that even O-type to early B-type stars can retain their dense, inner disks for 2-3 Myr at least. The latter fraction is an upper limit to the disk fraction at 2-3 Myr, however, as the study by Maercker \& Burton (2005) covers the extended star-forming region surrounding the central cluster, and objects along the star-forming ridges with ages younger than $1 \mathrm{Myr}$ are included in the $L$-band excess fraction. Despite being an upper limit, the 30 Dor disk fraction is significantly lower than expected from the disk fraction-age relation derived in nearby star-forming regions (Figure 13). As the $L$-band sample in 30 Dor is also dominated by early-type stars, the effect of the cluster environment and the disk host stars cannot be distinguished in this region.

At younger ages of 1-2 Myr, the massive star-forming regions NGC 3576, M17, and NGC 3603 provide templates at earlier evolutionary stages. In the more dispersed environment of NGC 3576, Maercker et al. (2006) find a cluster disk fraction of $55 \% \pm 2 \%$ with a strong decrease from $95 \% \pm 1 \%$ in the immediate cluster core to $27 \% \pm 5 \%$ at a cluster center distance of 6 pc. It is noteworthy that Figuerêdo et al. (2002) analyze the early B/late O-type star No. 48 in NGC 3576, and conclude on the basis of $\mathrm{CO}$ and $\mathrm{H}_{2}$ emission, along with the absence of stellar features, that the source is most likely a B1V star surrounded by a thick circumstellar disk or envelope, consistent with our interpretation of the Arches excess population. At an even younger age of less than $1 \mathrm{Myr}$, the young, massive cluster NGC 6618 at the center of the massive $\mathrm{H}$ II region M17 has recently been investigated in detail with regard to its $J H K L$ population. Hoffmeister et al. (2006) find an $L$-band excess fraction of $41 \%$ among a population of 201 M17 sources dominated by B-type stars. In medium-resolution spectroscopy, they find nine stars with early B-type luminosities with $\mathrm{CO}$ emission features, and seven or $78 \%$ of these $\mathrm{CO}$ emitters feature $L$-band excesses. Interestingly, two objects also show $\mathrm{Br} \gamma$ and $\mathrm{Pa} \delta$ in absorption. If we identify the M17 CO emission objects with the Arches $L^{\prime}$-excess population, and hence with B-type stars retaining optically thick gaseous disks, both the low fraction of $6 \% \pm 2 \% L^{\prime}$-band excess sources and the detection of $\mathrm{CO}$ emission in all three of our excess sources with spectra is consistent with a more evolved counterpart of the younger M17 population. The denser and more hostile environment of the Arches cluster additionally causes the disk fraction to be substantially depleted as compared to the younger, and less dense, environment of M17.

At an age of $1 \mathrm{Myr}$, the compact, massive cluster NGC 3603 YC is most similar to the Arches cluster in its core density and total mass (see, e.g., Stolte et al. 2006 for a detailed comparison of the cluster properties). A disk fraction of $27 \% \pm 3 \%$ was measured in the central $1 \mathrm{pc}$ for stars in the mass range $1 M_{\odot}<M<20 M_{\odot}$ (Stolte et al. 2004), and a lower fraction of $12 \% \mathrm{~L}$-band excess sources was observed among the main sequence $\mathrm{OB}$ star population in the radial range from 0.2 to $0.8 \mathrm{pc}$ from seeing-limited JHKL observations. Using highspatial resolution imaging, Harayama et al. (2008) recently resolved the central 0.4 pc of the NGC 3603 YC cluster core, and derived an $L$-band excess fraction of $24 \% \pm 10 \%$ for the entire population with $M>1 M_{\odot}$, and $22 \% \pm 10 \%$ for main sequence stars with $M>4 M_{\odot}$ only. The higher excess fraction in the cluster core is most likely a consequence of resolving the fainter excess population in the high angular resolution $L$-band observations. This disk fraction is substantially lower than the characteristic disk fraction of nearby 1-2 Myr starforming regions (Figure 13). In the disk fraction versus age diagram, the three densest clusters Arches, NGC 3603, and NGC 6618 (M17) display the lowest disk fractions in their respective age group. This is not an effect of the starburst cluster environment exclusively, as the disk fractions of Herbig $\mathrm{Ae} / \mathrm{Be}$ stars in IC 348 and $\sigma$ Ori are comparably low, suggesting that 
Table 7

Physical Properties and Disk Fractions of Young Star Clusters

\begin{tabular}{|c|c|c|c|c|c|c|c|c|c|c|}
\hline \multirow[t]{2}{*}{ Name } & \multirow[t]{2}{*}{ Location } & \multirow{2}{*}{$\begin{array}{l}\text { Dist } \\
(\mathrm{kpc})\end{array}$} & \multirow{2}{*}{$\begin{array}{l}\text { Age } \\
(\mathrm{Myr})\end{array}$} & \multirow{2}{*}{$\begin{array}{c}M_{\mathrm{cl}} \\
\left(M_{\odot}\right)\end{array}$} & \multirow{2}{*}{$\begin{array}{l}r_{\text {core }} \\
(\mathrm{pc})\end{array}$} & \multirow{2}{*}{$\begin{array}{c}\rho_{\text {core }} \\
\left(M_{\odot} \mathrm{pc}^{-3}\right)\end{array}$} & \multicolumn{2}{|c|}{ Disk Fraction } & \multirow{2}{*}{$\begin{array}{l}\text { Mass Range } \\
\qquad\left(M_{\odot}\right)\end{array}$} & \multirow[t]{2}{*}{ Ref. } \\
\hline & & & & & & & All & OB(A) Stars & & \\
\hline \multicolumn{11}{|c|}{ Starburst Clusters $M_{\mathrm{cl}}>10^{4} M_{\odot}$} \\
\hline Quintuplet & GC & 8 & $4 \pm 1$ & $2 \times 10^{4}$ & $\ldots$ & & & $\ldots$ & $\ldots$ & 5 \\
\hline NGC 3603 YC & SP & $6-7$ & 1 & $10^{4}$ & 0.2 & $\sim 10^{5}$ & $27 \pm 3$ & $22 \pm 10$ & $1.2-20 M_{\odot}$ & 6,7 \\
\hline Westerlund 1 & SP & $3.5 \pm 0.2$ & $4 \pm 1$ & 52000 & $<1$ & $>2 \times 10^{4}$ & $\ldots$ & $\ldots$ & $\ldots$ & 8 \\
\hline \multicolumn{11}{|c|}{ Young Clusters $M_{\mathrm{cl}}<10^{4} M_{\odot}$} \\
\hline ONC/Trap & SP & 0.43 & $0.3-1$ & $10^{3}$ & 0.2 & $410^{4}$ & 80 & 42 & $0.2-35 M_{\odot}$ & 11,12 \\
\hline NGC 2024 & SP & 0.46 & $0.3-1$ & 200 & $\ldots$ & $6 \times 10^{3}$ & $85 \pm 5$ & 58 & $0.1-20 M_{\odot}$ & 12 \\
\hline$\sigma$ Ori & SP & 0.5 & $2.5-3$ & 225 & 1.6 & 4 & $27 \pm 3$ & $4 \pm 4$ & $0.1-3 M_{\odot}$ & 13,14 \\
\hline NGC 3576/RCW 57 & SP & 2.8 & $1.5 \pm 1$ & $5 \times 10^{3}$ & $\ldots$ & $3 \times 10^{3}$ & $\ldots$ & $55 \pm 12$ & $10-35 M_{\odot}$ & 15,16 \\
\hline M17/NGC 6618 & SP & 2.1 & $0.3-1$ & $>10^{3}$ & $\ldots$ & $>60$ & 62 & 41 & $2-90 M_{\odot}$ & 17 \\
\hline
\end{tabular}

References. (1) Espinoza et al. 2009; (2) Stolte et al. 2005; (3) Najarro et al. 2004; (4) Figer et al. 1999; (5) Figer et al. 2002; (6) Harayama et al. 2008; (7) Stolte et al. 2004; (8) Brandner et al. 2008; (9) Brandl et al. 1996; (10) Maercker \& Burton 2005; (11) Hillenbrand \& Hartmann 1998; (12) Haisch et al. 2000, 2001; (13) Hernández et al. 2007; (14) Sherry et al. 2004; (15) Figuerêdo et al. 2002; (16) Maercker et al. 2006; (17) Hoffmeister et al. 2006; (18) Dahm \& Simon 2005; (19) Lada et al. 2006; (20) Lada \& Lada 1995; (21) Dahm 2005; (22) Dahm \& Hillenbrand 2007.

disk depletion is dominated by the stellar mass of the host star. The low disk fractions observed in all of these massive clusters among early-type stars is consistent with a more rapid depletion of disks around high-mass stars than lower-mass cluster members. There is increasing evidence that young, massive clusters comprised of a large population of OB-type stars have lower disk fractions than clusters dominated by low-mass stars. In Figure 13, the massive clusters Arches, NGC 3603, 30 Dor, NGC 3576, NGC 6618, as well as $\sigma$ Orionis all fall below the linear trend derived by Haisch et al. (2001). It is particularly striking that the four massive clusters where predominantly Btype stars are probed (including the Herbig Ae/Be data point for $\sigma$ Ori), appear to follow the same fractional decrease in the disk fraction with age shifted to lower total disk fractions.

We thus conclude that the $L^{\prime}$-band excess of the Arches sources is produced by remnants of massive accretion disks around mid to late B-type stars in a more evolved state than the $\mathrm{CO}$ and $\mathrm{Br} \gamma$ emission disks observed in the younger UCHII regions and star-forming clusters such as the Trapezium, M17, NGC 3576, and NGC 2024 with ages $\leqslant 1$ Myr.

\section{SUMMARY}

We report the detection of $L^{\prime}$-band excess sources in the GC Arches cluster. Out of 24 excess sources with $K^{\prime}-L^{\prime}>2.0 \mathrm{mag}$, all 21 sources with precise proper motion measurements are proper motion members of the cluster. The $\mathrm{CO}$ bandhead emission detected in three sources with $K$-band spectroscopy identifies these sources as optically thick disks. The comparison with disk simulations in similarly young star-forming regions suggests that the $\mathrm{CO}$ emission and $L^{\prime}$-band excess arise in the inner few $\mathrm{AU}$ from the central star. The $H$-band magnitude range of these sources indicates that the sample consists of Herbig Be stars, possibly with a small contribution of early Herbig Ae stars.

From the complete sample of proper motion members with $H<19 \mathrm{mag}$, we derive a disk fraction of $6 \% \pm 2 \%$ among
B-type stars in the central Arches cluster. At the young cluster age of $2.5 \mathrm{Myr}$, this disk fraction is significantly lower than expected from the disk fraction-age relation found in starforming regions with lower central density at ages 2-3 Myr. Even when compared to B-star samples in other massive starforming clusters at similar ages, such as 30 Dor or NGC 2264, the dense Arches and $\sigma$ Ori clusters feature the lowest disk fraction measured at this age. The disk fraction in the Arches cluster increases from $2.7 \% \pm 1.8 \%$ in the cluster core $(r<$ $0.16 \mathrm{pc})$ to $5.4 \% \pm 2.6 \%$ at intermediate radii $(0.16 \mathrm{pc}<r<$ $0.3 \mathrm{pc})$ and $9.7 \% \pm 3.7 \%$ outside the cluster center $(r>0.3 \mathrm{pc})$. The preferential depletion of disks in the cluster core evidences disk destruction by UV radiation, winds, and tidal encounters of massive stars. The comparison with the younger starburst cluster NGC 3603 and more moderate star-forming regions provides a strong observational indication that disks are more rapidly depleted in a starburst environment.

In the case of a very dense cluster such as the Arches, the detection of a significant population of circumstellar disks nevertheless comes as a surprise. In the presence of on the order of 125 O-type stars contributing to the intense cluster radiation field, which ionizes the surrounding molecular clouds (Lang et al. 2001), disk destruction must be very efficient. The survival of disks over several Myr in such a dense cluster environment sheds new light on the emergence of debris disks around high-mass stars, and the possibility of forming, and detecting, planetary systems in dense, massive clusters.

Combining the lower total disk fraction observed in dense, massive clusters with the depletion of disks in the core of the Arches cluster as compared to larger radii (Section 3.3.2), provides strong indication that clusters with substantial OB star populations have lower disk fractions especially in the immediate environment of high-mass stars. These results suggest that the rate of disk depletion and thus the disk lifetime depend not only on the spectral type of the disk host stars, but also on the total cluster mass and density as an indicator for the ambient 


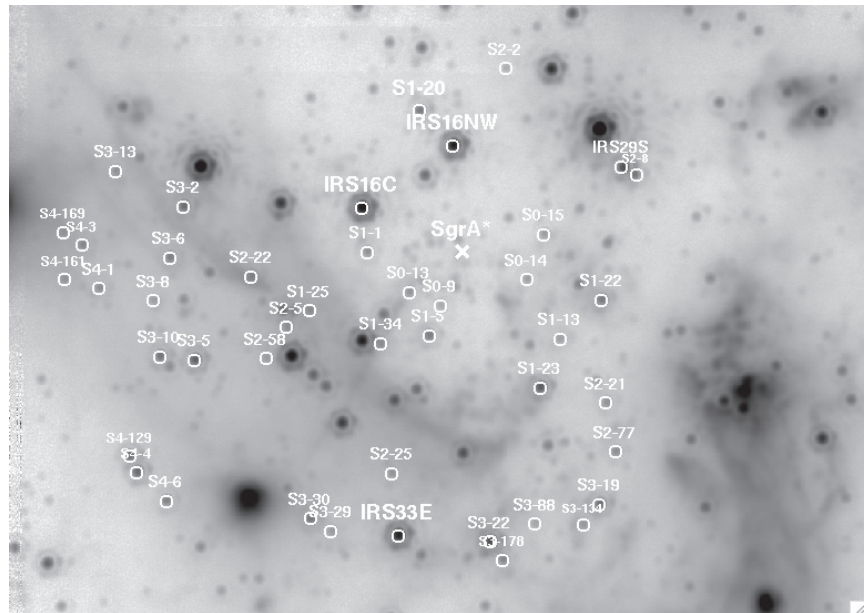

Figure A.1. Finding chart of non-variable $L^{\prime}$ calibration sources located in the Galactic center (north is up, east is to the left).

UV radiation field and the frequency of dynamical interactions with the massive OB stars specifically in the cluster cores.

This work would not have been possible without the intense effort and dedication of the Keck LGS-AO staff. We are deeply grateful for their support enabling these observations. The W. M. Keck Observatory is operated as a scientific partnership among the California Institute of Technology, the University of California, and the National Aeronautics and Space Administration. The Observatory was made possible by the generous financial support of the W. M. Keck Foundation. The authors wish to recognize and acknowledge the very significant cultural role and reverence that the summit of Mauna Kea has always had within the indigenous Hawaiian community. We are most fortunate to have the opportunity to conduct observations from this mountain. A.S. acknowledges support by the German Science Foundation Emmy Noether program (STO 496/3-1). This work was supported by NSF grant AST 04-06816 and by the Science and Technology Center for Adaptive Optics, managed by the University of California at Santa Cruz under cooperative agreement AST 98-76783.

Facilities: Keck:II (NIRC2), VLT:Yepun

\section{APPENDIX A}

\section{GALACTIC CENTER $L^{\prime}$ CALIBRATION SOURCES}

The absolute zero point of the Arches core field $L^{\prime}$ observations was derived by observing the central $10^{\prime \prime}(0.4 \mathrm{pc})$ around Sgr A during the same night as the cluster center. Detailed descriptions of the observing strategy can be found in Ghez et al. (2005), Hornstein et al. (2007), and Lu et al. (2009). Table A.1 lists the non-variable GC sources used as calibrators for the absolute $L^{\prime}$ zero point in the GC. These sources are identified in Figure A.1 (see also Lu et al. 2009 for the naming convention). The zero-point calibration is detailed in Section 2.1.3. The foreground extinction to the central parsec, $A_{V} \sim 26-36 \mathrm{mag}$, is comparable to the foreground extinction toward the Arches. The advantage of this central reference field is that there are numerous calibration sources with comparably red colors as the Arches cluster stars.

Figure A.2 shows the zero point derived from the combined image of 19 frames taken in the GC observing sequence. The combined image yields a zero point of $\mathrm{ZPT}_{L^{\prime}}=23.196 \pm$
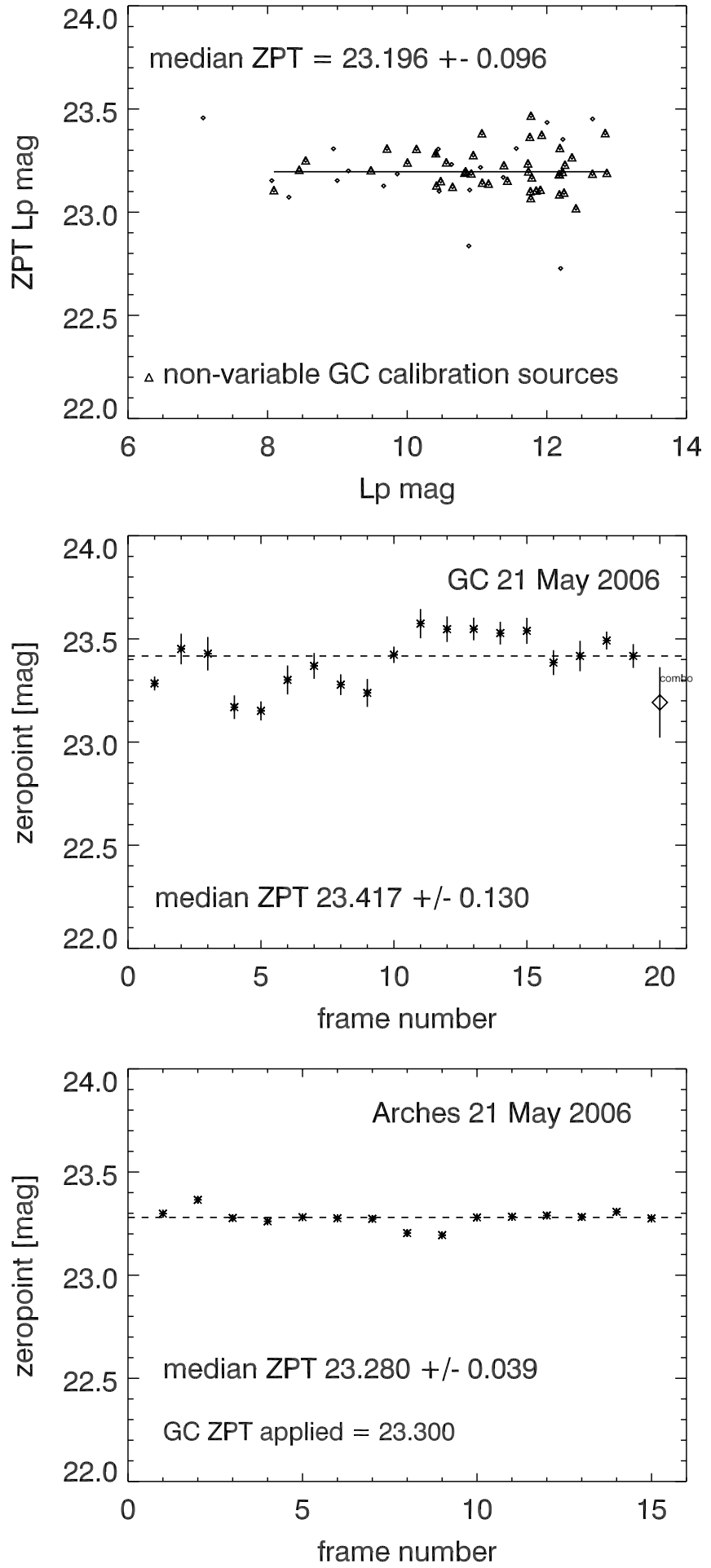

Figure A.2. Zero-point determination of GC sources and variations throughout the observing sequence. Upper panel: $L^{\prime}$ zero point of non-variable sources in the Galactic center (GC), derived from reference magnitudes provided in Table A.1. This zero point was derived from the combined image composed of 19 frames. Middle panel: zero-point variation between individual GC frames from the GC $L^{\prime}$ observing sequence. Bottom panel: zero-point variations between individual Arches frames as observed on the core field, after a GC zero point of 23.300 has been applied.

$0.096 \mathrm{mag}$, while the individual frames yield a median zero point of $\mathrm{ZPT}_{L^{\prime}}=23.417 \pm 0.130 \mathrm{mag}$. The zero-point variations among the Arches individual images are significantly smaller with a standard deviation of only $0.039 \mathrm{mag}$, indicating that 
Table A.1

$L^{\prime}$ Calibration Sources in the Galactic Center

\begin{tabular}{|c|c|c|c|c|c|c|c|c|c|}
\hline Name & Radius (") & $\delta$ R.A. & $\delta$ Decl. & $H \operatorname{Ref}^{\mathrm{a}}$ & $K^{\prime} \operatorname{Ref}^{\mathrm{a}}$ & $L^{\prime} \operatorname{Ref}^{\mathrm{a}}$ & $H \mathrm{Cal}^{\mathrm{b}}$ & $K^{\prime} \mathrm{Cal}^{\mathrm{b}}$ & $L^{\prime} \mathrm{Cal}^{\mathrm{b}}$ \\
\hline IRS16C & 1.209 & 1.112 & 0.476 & 11.940 & 9.792 & 8.090 & 11.945 & 9.952 & 8.182 \\
\hline IRS16NW & 1.178 & 0.000 & 1.178 & 12.080 & 10.061 & 8.452 & 12.080 & 10.197 & 8.445 \\
\hline IRS33E & 3.121 & 0.673 & -3.047 & 12.260 & 10.948 & 8.545 & 12.136 & 10.269 & 8.492 \\
\hline IRS29S & 2.028 & -1.818 & 0.901 & 13.310 & 11.262 & 9.710 & 13.579 & 11.338 & 9.600 \\
\hline $\mathrm{S} 1-23$ & 1.701 & -0.896 & -1.446 & 13.980 & 11.854 & 10.003 & 13.861 & 11.743 & 9.961 \\
\hline S3-2 & 3.036 & 2.981 & 0.575 & 14.060 & 12.027 & 10.480 & 13.874 & 12.111 & 10.528 \\
\hline $\mathrm{S} 2-8$ & 2.109 & -1.949 & 0.806 & 13.770 & 12.148 & 11.070 & 13.971 & 12.223 & 10.886 \\
\hline S3-5 & 3.106 & 2.896 & -1.123 & 14.430 & 12.140 & 10.132 & 14.246 & 12.032 & 10.024 \\
\hline S3-10 & 3.471 & 3.299 & -1.080 & 13.610 & 12.327 & 10.650 & 13.699 & 12.116 & 10.725 \\
\hline S3-19 & 3.169 & -1.557 & -2.760 & 13.890 & 12.470 & 10.560 & 13.863 & 12.018 & 10.517 \\
\hline $\mathrm{S} 1-20$ & 1.615 & 0.372 & 1.571 & 15.000 & 12.596 & 10.822 & 15.102 & 12.765 & 10.829 \\
\hline $\mathrm{S} 1-22$ & 1.686 & -1.608 & -0.508 & 14.650 & 12.693 & 10.947 & 14.657 & 12.646 & 10.868 \\
\hline S4-129 & 4.224 & 3.633 & -2.155 & $\ldots$ & 12.744 & 10.420 & 14.468 & 12.208 & 10.489 \\
\hline S1-5 & 0.979 & 0.368 & -0.907 & 14.930 & 12.727 & 10.918 & 14.771 & 12.640 & 10.928 \\
\hline S3-30 & 3.343 & 1.690 & -2.884 & 14.540 & 12.992 & 10.837 & 14.404 & 12.433 & 10.838 \\
\hline S4-6 & 4.186 & 3.245 & -2.644 & 15.580 & 13.370 & 11.165 & 15.050 & 12.852 & 11.225 \\
\hline S3-6 & 3.165 & 3.164 & 0.080 & 14.890 & 12.827 & 11.075 & 14.991 & 12.818 & 11.130 \\
\hline $\mathrm{S} 2-22$ & 2.294 & 2.282 & -0.228 & 14.740 & 12.881 & 10.882 & 14.641 & 12.888 & 11.245 \\
\hline $\mathrm{S} 1-34$ & 1.273 & 0.848 & -0.949 & 14.770 & 13.013 & 11.773 & 14.515 & 13.069 & 11.503 \\
\hline S1-1 & 0.965 & 0.965 & 0.033 & 14.930 & 13.052 & 11.730 & 14.843 & 13.130 & 11.692 \\
\hline $\mathrm{S} 2-5$ & 2.022 & 1.860 & -0.793 & 15.170 & 13.231 & 11.770 & 15.250 & 13.312 & 11.900 \\
\hline S0-13 & 0.686 & 0.537 & -0.427 & 15.430 & 13.408 & 11.765 & 15.375 & 13.407 & 11.861 \\
\hline $\mathrm{S} 1-25$ & 1.733 & 1.625 & -0.600 & 15.470 & 13.466 & 11.785 & 15.409 & 13.366 & 11.815 \\
\hline $\mathrm{S} 2-21$ & 2.322 & -1.656 & -1.627 & 15.380 & 13.526 & 11.927 & 15.394 & 13.445 & 11.750 \\
\hline S0-14 & 0.807 & -0.756 & -0.282 & 15.520 & 13.656 & 12.360 & 15.662 & 13.763 & 12.292 \\
\hline S0-15 & 0.946 & -0.906 & 0.272 & 15.620 & 13.660 & 12.183 & 15.678 & 13.732 & 12.198 \\
\hline S2-77 & 2.754 & -1.705 & -2.163 & 15.420 & 13.857 & 11.757 & 15.227 & 13.603 & 11.589 \\
\hline S3-29 & 3.332 & 1.442 & -3.004 & 15.480 & 14.079 & 12.225 & 15.571 & 13.628 & 12.228 \\
\hline S2-25 & 2.508 & 0.763 & -2.389 & 15.880 & 14.106 & 12.175 & 15.952 & 13.820 & 12.189 \\
\hline S2-58 & 2.417 & 2.135 & -1.133 & 16.000 & 14.079 & 12.418 & 16.013 & 14.084 & 12.597 \\
\hline S2-2 & 2.060 & -0.569 & 1.980 & 15.460 & 14.090 & 12.860 & 15.828 & 14.052 & 12.868 \\
\hline S1-13 & 1.392 & -1.069 & -0.891 & 16.380 & 14.180 & 12.247 & 16.126 & 14.050 & 12.350 \\
\hline S3-8 & 3.388 & 3.362 & -0.421 & 15.630 & 14.014 & 12.180 & 15.654 & 13.874 & 12.292 \\
\hline S0-9 & 0.554 & 0.143 & -0.535 & 16.150 & 14.348 & 12.837 & 16.002 & 14.173 & 12.652 \\
\hline S3-22 & 3.157 & -0.316 & -3.142 & 13.260 & 11.671 & 9.480 & 13.133 & 11.167 & 9.475 \\
\hline S4-3 & 4.104 & 4.100 & 0.172 & 14.870 & 13.223 & 11.435 & 14.874 & 13.249 & 11.481 \\
\hline S3-178 & 3.410 & -0.395 & -3.387 & 15.220 & 13.467 & 11.385 & 15.098 & 13.067 & 11.356 \\
\hline S4-169 & 4.360 & 4.350 & 0.299 & 15.230 & 13.674 & 12.260 & 15.313 & 13.881 & 12.228 \\
\hline S4-1 & 3.987 & 3.976 & -0.299 & 15.400 & 13.173 & 11.737 & 15.442 & 13.376 & 11.737 \\
\hline S4-161 & 4.338 & 4.333 & -0.207 & 15.840 & 13.897 & 11.907 & 15.893 & 13.926 & 11.997 \\
\hline S3-134 & 3.272 & -1.329 & -2.991 & 16.080 & 13.807 & 12.187 & 16.045 & 13.848 & 12.074 \\
\hline S3-88 & 3.095 & -0.802 & -2.989 & 16.480 & 14.466 & 12.653 & 16.056 & 14.324 & 12.665 \\
\hline S4-4 & 4.216 & 3.560 & -2.260 & $\ldots$ & 12.793 & 10.410 & 13.937 & 11.965 & 10.323 \\
\hline S3-13 & 3.843 & 3.729 & 0.927 & $\ldots$ & 13.578 & 11.847 & 15.855 & 13.566 & 11.940 \\
\hline
\end{tabular}

Notes.

a Columns 5-7 refer to reference magnitudes from earlier NGSAO observations in the central parsec, calibrated against photometry in Clénet et al. (2001, 2004) and Blum et al. (1996).

b Columns 8-10 refer to photometry taken on 2006 May 21, and calibrated with respect to the reference magnitudes presented in Columns 5-7. A comparison of $L^{\prime}$ calibrated and reference magnitudes is shown in Figure A.2.

atmospheric conditions had equilibrated when the cluster was observed. Hence, a mean zero point between the combined GC image and the individual frames of 23.300 was applied to the combined Arches core image. The zero-point uncertainty is estimated to be $\sim 0.10 \mathrm{mag}$ due to atmospheric/temperature variations during the observing sequence.

\section{APPENDIX B}

\section{ARCHES $L^{\prime}$ ZERO-POINT CALIBRATION AND $L$-BAND EXTINCTION NOTES}

The Arches $L$-band observations were obtained with the Mauna Kea $L$ ' filter. While the GC high-resolution observations by Clénet et al. (2001) were obtained with the similar VLT $L^{\prime}$ filter $\left(\lambda_{c}=3.8 \mu \mathrm{m}, \Delta \lambda=0.62 \mu \mathrm{m}\right)$, earlier GC observations from Blum et al. (1996) used as calibration reference also by Clénet et al. (2001) were observed with the obsolete $L$ filter, which has a comparable width but lower central wavelength than $L^{\prime}\left(\lambda_{c}=3.45 \mu \mathrm{m}\right.$, DePoy \& Sharp 1991; see also Simons $\&$ Tokunaga 2002 for filter definitions). Earlier extinction laws toward the central galaxy by Rieke \& Lebofsky (1985) and Mathis (1990) were determined from the obsolete $L$ filter, while newer determinations make use of the Spitzer database, employing the IRAC $\lambda_{c}=3.6 \mu \mathrm{m}$ band. Extrapolation of the standard extinction law reviewed in Mathis (1990) with a slope of $\alpha=1.70$ yields $A_{L} / A_{V}=0.058$ versus $A_{L^{\prime}} / A_{V}=0.043$. 
The difference for the $K^{\prime}$ band, on the other hand, is with $A_{K} / A_{V}=0.112$ versus $A_{K^{\prime}} / A_{V}=0.114$ negligible, and the $H$-band central wavelength and bandwidth did not change significantly.

$A_{V}$ values of the Arches $L^{\prime}$-excess sources span a range from 23 to $28 \mathrm{mag}$ with an average of $25.3 \mathrm{mag}$ (Table 6). These extinctions were derived from the four nearest cluster members to each excess source, and are representative of the cluster population. With the above values for $L$-band extinction, we expect stars with $A_{V} \sim 23 \mathrm{mag}$ to be brighter in $L^{\prime}$ by $\Delta\left(L-L^{\prime}\right)=(0.058-0.043) A_{V}=0.34 \mathrm{mag}$ with respect to $L$, and sources with $A_{V} \sim 28$ mag brighter in $L^{\prime}$ by $0.42 \mathrm{mag}$. The mean offset between the $L$ and $L^{\prime}$ filters for the median extinction of the GC calibration sources, $\Delta\left(L-L^{\prime}\right)=0.50$ for $A_{V, \mathrm{GC}}=33.1 \mathrm{mag}$, is corrected by calibrating the Clénet et al. (2001) $L^{\prime}$ measurement with Blum et al. (1996) $L$-band magnitudes. The residual, extinction dependent offset expected for Arches cluster members with $A_{V \text {, Arches }}=25.3 \mathrm{mag}$ and $\Delta\left(L-L^{\prime}\right)=0.38 \mathrm{mag}$ is therefore $\Delta\left(L-L^{\prime}\right)_{G C}-\Delta(L-$ $\left.L^{\prime}\right)_{\text {Arches }}=0.50-0.38=0.12 \mathrm{mag}$, which explains the offset of Arches cluster members from the standard extinction law in the two-color diagram, as discussed in Section 2.1.3. The range in extinctions in the cluster population $22.7 \mathrm{mag}$ $<A_{V}<27.9$ mag corresponds to $0.43-0.34=0.08 \mathrm{mag}$, which is within our uncertainties of $\sigma_{L^{\prime}} \sim 0.1 \mathrm{mag}$. This offset would appear as a bend in the reddening track in the two-color diagram (see Stead \& Hoare 2009 for a discussion on nonstraight reddening tracks). This bend is not detectable given the photometric uncertainties discussed in Section 2.1.

Note that the $L$-band calibration from GC sources, where the bulk offset between $L$ and $L^{\prime}$ was corrected, implies that the standard $L$-band extinction law represents the proper extinction value of $A_{L} / A_{V}=0.058$, which has been used accordingly. The value of $A_{L^{\prime}} / A_{V}=0.043$ extrapolated from the central wavelength of the $L^{\prime}$ filter yields a reddening track that is too flat given this $L$-band calibration procedure.

\section{REFERENCES}

Alonso-Albi, T., et al. 2009, A\&A, 497, 117

Alves, D. R. 2000, ApJ, 539, 732

Armitage, P. J. 2000, A\&A, 362, 968

Bik, A., Kaper, L., Hanson, M. M., \& Smits, M. 2005, A\&A, 440, 121

Bik, A., Kaper, L., \& Waters, L. B. F. M. 2006, A\&A, 455, 561

Bik, A., \& Thi, W. F. 2004, A\&AL, 427, 13

Blum, R. D., Sellgren, K., \& Depoy, D. L. 1996, ApJ, 470, 864

Blum, R. D., et al. 2001, AJ, 122, 1875

Blum, R. D., et al. 2004, ApJ, 617, 1167

Boffin, H. M. J., et al. 1998, MNRAS, 300, 1189

Brandl, B., et al. 1996, ApJ, 466, 254

Brandner, W., et al. 2008, A\&A, 478, 137

Carpenter, J. M. 2001, AJ, 121, 2851

Carr, J. S., Tokunaga, A. T., Najita, J., Shu, F. H., \& Glassgold, A. E. 1993, ApJ, 411, L37

Cesaroni, R., et al. 2007, in Protostars \& Planets V, ed. B. Reipurth, D. Jewitt, \& K. Keil (Tucson, AZ: Univ. Arizona Press), 197

Chandler, C. J., Carlstrom, J. E., \& Scoville, N. Z. 1995, ApJ, 446, 793

Clénet, Y., Rouan, D., Gendron, E., Montri, J., Rigaut, F., Léna, P., \& Lacombe, F. 2001, A\&A, 376, 124

Clénet, Y., et al. 2004, A\&A, 417, L15

Cotera, A. S., et al. 1996, ApJ, 461, 750
Crowther, P. A. 2007, ARA\&A, 45, 177

Dahm, S. E. 2005, AJ, 130, 1805

Dahm, S. E., \& Hillenbrand, L. A. 2007, AJ, 133, 2027

Dahm, S. E., \& Simon, T. 2005, AJ, 129, 829

DePoy, D. A., \& Sharp, N. L. 1991, AJ, 101, 1324

Diolaiti, E., et al. 2000, A\&AS, 147, 335

Espinoza, P., Selman, F. J., \& Melnick, J. 2009, A\&A, 501, 563

Fatuzzo, M., \& Adams, F. C. 2008, ApJ, 675, 1361

Figer, D. F., et al. 1999, ApJ, 525, 750

Figer, D. F., et al. 2002, ApJ, 581, 258

Figuerêdo, E., Blum, R. D., Daminelli, A., \& Conti, P. S. 2002, AJ, 124, 2739

Figuerêdo, E., Blum, R. D., Damineli, A., \& Conti, P. S. 2005, AJ, 129, 1523

Fruchter, A. S., \& Hook, R. N. 2002, PASP, 114, 144

Ghez, A. M., et al. 2005, ApJ, 635, 1087

Ghez, A. M. G., et al. 2008, ApJ, 689, 1044

Haisch, K. E., Jr., Lada, E. A., \& Lada, C. J. 2000, AJ, 120, 1396

Haisch, K. E., Jr., Lada, E. A., \& Lada, C. J. 2001, ApJ, 553, L153

Hanson, M. M., Conti, P. S., \& Rieke, M. J. 1996, ApJS, 107, 281

Hanson, M. M., Howarth, I. D., \& Conti, P. S. 1997, ApJ, 489, 698

Harayama, Y., Eisenhauer, F., \& Martins, F. 2008, ApJ, 675, 1319

Hawarden, T. G., et al. 2001, MNRAS, 325, 563

Hernández, J., et al. 2005, AJ, 129, 856

Hernández, J., et al. 2007, ApJ, 662, 1067

Hillenbrand, L. A. 1997, AJ, 113, 1733

Hillenbrand, L. A., \& Hartmann, L. W. 1998, ApJ, 492, 540

Hillenbrand, L. A., Strom, S. E., Vrba, F. J., \& Keene, J. 1992, ApJ, 397, 613

Hoffmeister, V. H., et al. 2006, A\&A, 457, L29

Hollenbach, D. J., Yorke, H. W., \& Johnstone, D. 2000, in Protostars and Planets

IV, ed. V. Mannings, A. P. Boss, \& S. S. Russell (Tucson, AZ: Univ. Arizona Press), 401

Hornstein, S. D., et al. 2007, ApJ, 667, 900

Kim, S. S., Figer, D. F., Kudritzki, R. P., \& Najarro, F. 2006, ApJ, 653, L113

Kraus, M., Krügel, E., Thum, C., \& Geballe, T. R. 2000, A\&A, 36, 158

Lada, E. A., \& Lada, C. J. 1995, AJ, 109, 1682

Lada, C. J., et al. 2000, AJ, 120, 3162

Lada, C. J., et al. 2006, AJ, 131, 1574

Lang, Cornelia C., Goss, W. M., \& Morris, M. 2001, AJ, 121, 2681

Lejeune, T., \& Schaerer, D. 2001, A\&A, 366, 538

Lu, J. R. 2008, PhD thesis, UCLA

Lu, J. R., et al. 2009, ApJ, 619, 1463

Maercker, M., \& Burton, M. G. 2005, A\&A, 438, 663

Maercker, M., Burton, M. G., \& Wright, C. M. 2006, A\&A, 450, 253

Martins, F., et al. 2008, A\&A, 478, 219

Mathis, J. S. 1990, ARA\&A, 28, 37

Meyer, M. R., Calvet, N., \& Hillenbrand, L. A. 1997, AJ, 114, 288

Najarro, F., Figer, D. F., Hillier, D. J., \& Kudritzki, R. P. 2004, ApJ, 611, L105

Natta, A., et al. 2001, A\&A, 371, 186

Olczak, C., Pfalzner, S., \& Eckart, A. 2008, A\&A, 488, 191

Pfalzner, S., Olczak, C., \& Eckart, A. 2006, A\&A, 454, 811

Richling, S., \& Yorke, H. W. 1997, A\&A, 327, 317

Richling, S., \& Yorke, H. W. 2000, ApJ, 539, 258

Rieke, G. H., \& Lebofsky, M. J. 1985, ApJ, 288, 618

Scally, A., \& Clarke, C. 2001, MNRAS, 325, 449

Sherry, W. H., Walter, F. M., \& Wolk, S. J. 2004, AJ, 128, 2316

Simons, D. A., \& Tokunaga, A. 2002, PASP, 114, 169

Stead, J. J., \& Hoare, M. G. 2009, MNRAS, 400, 731

Stolte, A., Brandner, W., Brandl, B., \& Zinnecker, H. 2006, AJ, 132, 253

Stolte, A., Brandner, W., Grebel, E. K., Lenzen, R., \& Lagrange, A.-M. 2005, ApJ, 628, L113

Stolte, A., Grebel, E. K., Brandner, W., \& Figer, D. F. 2002, A\&A, 394, 459

Stolte, A., et al. 2004, AJ, 128, 765

Stolte, A., et al. 2008, ApJ, 675, 1278

Takeuchi, T., Clarke, C. J., \& Lin, D. N. C. 2005, ApJ, 627, 286

Thompson, D., Egami, E., \& Sawicki, M. 2001, NIRC2 Pre-ship Review, available at http://www2.keck.hawaii.edu/inst/nirc2/preship_testing.pdf

van Dam, M. A., et al. 2006, PASP, 118, 310

Viehmann, T., et al. 2005, A\&A, 433, 117

Wizinowich, P. L., et al. 2006, PASP, 118, 297

Zinnecker, H., \& Yorke, H. W. 2007, ARA\&A, 45, 481 